\title{
The Demographics of Exoplanets
}

\author{
B. Scott Gaudi ${ }^{1}$, Jessie L. Christiansen ${ }^{2}$, and Michael R. Meyer ${ }^{3}$ \\ ${ }^{1}$ Department of Astronomy, The Ohio State University \\ ${ }^{2}$ Caltech/IPAC-NASA Exoplanet Science Institute, Pasadena CA \\ ${ }^{3}$ Department of Astronomy, University of Michigan
}

\section{Introduction}

The field of exoplanets can be thought of as resting on three broad pillars: demographics, characterization, and the search for habitable worlds and lif£ $\oint^{1}$ Of course, these divisions are somewhat arbitrary, the boundaries between them are generally fuzzy, and all three are interrelated. Nevertheless, subdividing the field into these three categories of inquiry provides a useful way to frame the science goals and questions of the exoplanet field. In the broadest sense, the overall goal of exoplanet demographic surveys is to determine the frequency and distribution of planets as a function of as many of the physical parameters that may influence planet formation and evolution as possible, over as broad of a range of these parameters as possible. These parameters can include, but are not limited to: 1) properties of the planets, including the planet mass $m_{p}$, radius $r_{p}$, and orbital properties (e.g., semimajor axis $a$, period $P$, eccentricity $e$ ); 2 ) properties of the host stars, including mass $M_{*}$, radius $R_{*}$, effective temperature $T_{\text {eff }}$, luminosity $L_{*}$, metallicity $[\mathrm{Fe} / \mathrm{H}]$ and detailed elemental abundances, activity, and age; and 3) environmental properties of the system, including multiplicity and birth environment.

These distribution functions represent the ultimate empirical data set (e.g., the "ground truth") that all theories of planet formation and evolution must reproduce. To the extent that most planets form in a bottom-up scenario, ab initio theories of planet formation must explain how the roughly micron-sized dust grains grow by $\sim 12-14$ orders of magnitude in radius and $\sim 38-40$ orders of magnitude in mass to the exoplanets we detect today. The physical mechanisms by which protoplanets accumulate mass and migrate as they cross through these many orders of magnitude in mass and radius vary dramatically, and may depend on the properties and environment of the host star, either directly or indirectly (see [110] for a review). The net result is that signatures of these physical mechanisms should be imprinted on these planet distribution functions ${ }^{2}$. Therefore, by comparing these planet distributions to the predictions of planet formation theories, we can begin to both test and refine these theories. An understanding of exoplanet demographics and the physical mechanisms that sculpted the planet distribution function is also essential for understanding planetary habitability. Of course, the first step is to determine the frequency of potentially habitable planets, often referred to as $\eta_{\oplus}{ }^{3}$. We discuss the most recent estimates of $\eta_{\oplus}$ and the opportunities for refining this estimate below. However, the habitability of any given planet can only be understood in the context of the entire planetary system, including its evolution. This is (in part) because classical theories predicted that planets that formed in the traditional habitable zone interior to the snow line of the disk were initially dry, thereby requiring giant planets to serve as a delivery mechanism for volatile species (e.g. carbon, nitrogen, and oxygen in the form of water) from beyond the snow line [91. On the other hand, more

\footnotetext{
${ }^{1}$ Although the study of disks is obviously strongly linked to the study of exoplanets, particularly because the properties of protoplanetary disks provide the ab initio conditions for planet formation, we will restrict our discussion in this short essay to exoplanets in systems where the planets are fully formed and the protoplanetary disk is essentially gone (e.g., ages of $\gtrsim 10 \mathrm{Myr}$, c.f. [136])

${ }^{2}$ The extent to which this is true may depend on the number of independent variables that contribute to the outcomes (e.g. the central limit theorem resulting in log-normal distributions), and which are scale free (e.g. resulting in power-law distributions).

${ }^{3}$ While there is no universally agreed-upon definition of $\eta_{\oplus}$, it generally refers to the frequency of terrestrial (rocky) planets in the traditional habitable zone of sunlike (FGK) stars. See, e.g., 66, 67.
} 

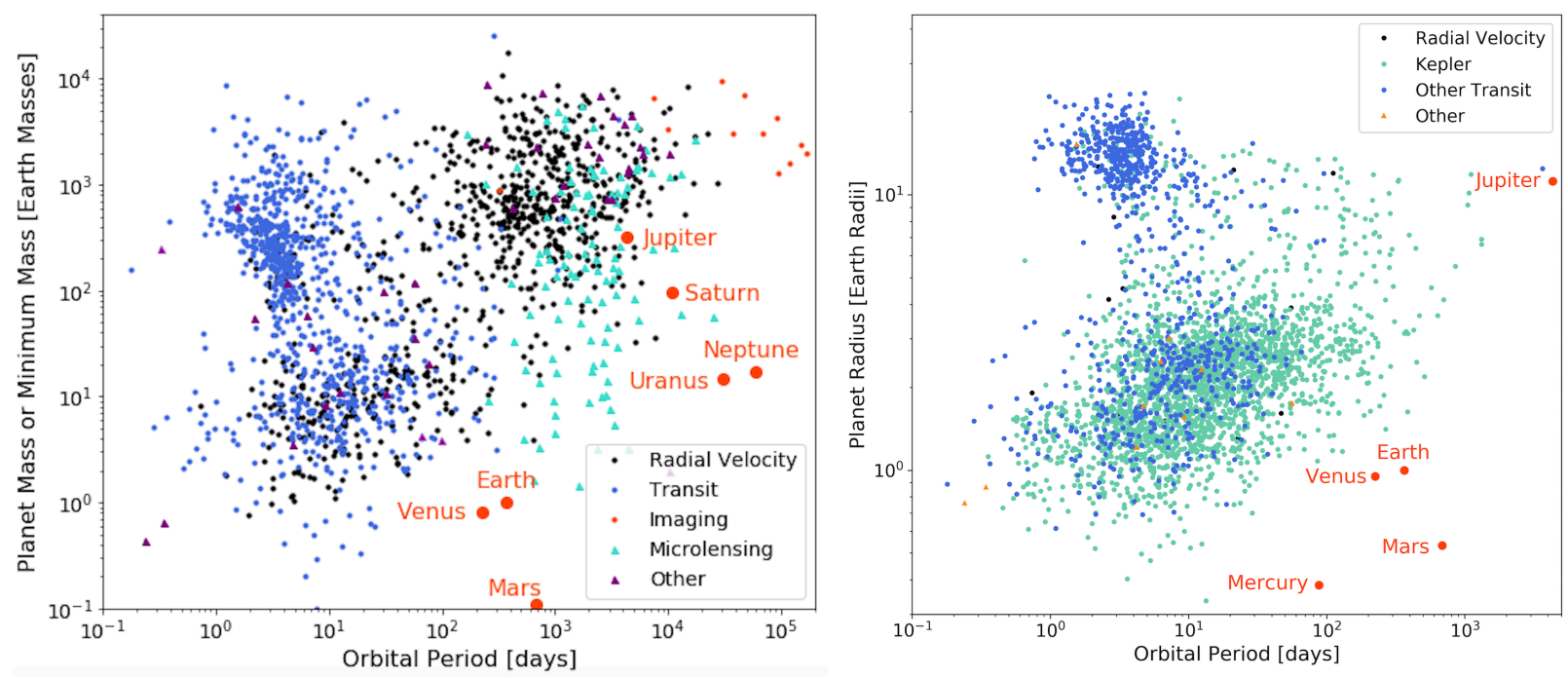

Figure 1: The distributions of the $\sim 4300$ confirmed low mass companions to stars as a function of their mass and period (left) and radius and period (right) where semi-major axis is converted to period Kepler's third law. The color coding denotes the method by which planets were detected. Some of the features in these diagrams are real, however many are due to the selection effects. For example, the large population of 1-4 $R_{\oplus}$ planets in the right panel is largely missing in the left panel due to the fact that these planets were primarily detected by Kepler, and thus typically have host stars that are too faint to enable a measurement of their mass via radial velocity. Similarly, the fact that there are nearly equal numbers of Hot Jupiters as cold Jupiters in the left panel is due to the fact that ground-based transit surveys, which have larger sample sizes than radial velocity surveys, are primarily sensitive to Hot Jupiters. Thus Hot Jupiters are over-represented in the figures. Finally, the paucity of planets in the lower-right corner of each plot is purely a selection effect due to the fact that the radial velocity, transit, and direct imaging methods are not currently sensitive to planets in this region of parameter space. Note that $\sim 25$ directly-imaged planets are not shown in the left plot because they have periods that are greater than $\gtrsim 10^{6}$ days. This figure is based on data from the NASA Exoplanet Archive: https://exoplanetarchive.ipac.caltech.edu/.

recent theories predict that water rich "pebbles" are likely to migrate interior to the snow line, which may result in the formation of many water worlds in planet population synthesis models (c.f. [73]). Thus giant planets formed near the snow line could also act barriers to limit the inward migration of such volatile-rich material.

\section{State of the art}

Although it was not the first exoplanet to be detected [22, 71, 135, the discovery of the Jovian companion to 51 Pegasi by 85 ignited the field of exoplanets. There are now over 4300 confirmed exoplanet ${ }^{4}$ which were primarily detected with four techniques: radial velocities, transits, microlensing, and direct imaging. The distribution of the properties of known exoplanets in mass/period and radius/period space is shown in Figure 1 .

Essentially all of the initial discoveries were made by the radial velocity (RV) method. For many years, the primary focus of the field was on discovering new exoplanets and improving the precision of RV surveys in order to expand the region of parameter space to which the method was sensitive. Eventually, the sample of known exoplanets detected in individual RV surveys was sufficiently large that a reasonably constraining statistical analysis could be performed. The first such statistical analysis was that of the Lick planet survey by [29], which contained 74 stars hosting 8 confirmed planetary companions, and several additional candidates.

\footnotetext{
${ }^{4}$ As of 11/23/2020; https://exoplanetarchive.ipac.caltech.edu/
} 
These results were superseded by the analysis of the Keck RV survey by [28, which included 585 stars hosting 48 planets. They fit a power-law mass-period distribution for planets with minimum mass $m_{p} \sin i>0.3 M_{\text {Jup }}$ and $P<2000$ days, finding

$$
\frac{d N}{d \ln m_{p} \sin i d \ln P} \propto\left(m_{p} \sin i\right)^{-0.31 \pm 0.20} P^{0.26 \pm 0.10}
$$

with a normalization factor such that $\sim 10 \%$ of solar-type stars host at least one planet with mass and period in the above range. Extrapolating, they estimated that $\sim 20 \%$ of solar-type stars host a giant planet (>0.3 $M_{\text {Jup }}$ within 20 au. In the interim, several authors made the first explorations of the dependence of giant planet frequency on host star mass and metallicity (e.g., [42, 36, 60, 58,).

The precision of RV surveys has improved to the point that they are now sensitive to planets with mass as low as a few Earth masses with relatively short $(P \lesssim 50$ days $)$ periods. Furthermore, the time baseline of some of the longest-running surveys is such that they are now becoming sensitive to Jovian planets with periods approaching 30 years. Some of the most recent statistical analyses of RV surveys can be found in [54, 57, 84, 13, 34, 39]. The primary conclusions of these studies are broadly consistent and can be roughly summarized as: (1) For relatively short periods and solar-type hosts (setting aside hot Jupiters), the planet mass function rises dramatically towards low-mass planets, such that sub-Neptunes and super-Earths are at least an order of magnitude more common than giant planets in the same period range; (2) The frequency of giant planets orbiting solar-type stars increases with increasing period (in agreement with [28]) up to $\sim 5$ years $(\sim 3 \mathrm{au})$, or roughly the location of the snow line in the solar protoplanetary disk [91, and then appears to decline with increasing separation; (3) the occurrence rate for giant planets with semimajor axes of $\lesssim 2.5$ au increases with host star mass and metallicity [36, 58.

Simultaneously, surveys using other exoplanet detection methods were being developed and conducted, including the transit, microlensing, and direct imaging methods. These surveys yielded the first detections of exoplanets by transits in 2003 [124, 63]), by microlensing in 2004 [12, and by direct imaging in 2008 81 $5^{5}$ Eventually, these methods also accrued sufficiently large samples of exoplanets to perform meaningful statistical analysis, see, e.g. [42, 47, 23, 48, 90, 20, 50, 82, 87.

To date, microlensing surveys have detected over 100 exoplanets. The most state-of-the art statistical analysis of the frequency of bound planets detected by microlensing was performed by [120], based on the Microlensing Observations in Astrophysics II survey (MOA-II). Their sample consisted of 23 planet detections. Microlensing is generally sensitive to the planet/star mass ratio $q=m_{p} / M_{*}$ rather than the planet mas: ${ }^{6}$. In addition, it is sensitive to the instantaneous projected separation of the planet in units of the angular Einstein ring radius $s$. Microlensing is generally sensitive to planets orbiting a range of hosts, including brown dwarfs, main-sequence stars, and stellar remnants. Although the majority of hosts are expected to be low-mass stars, a significant fraction will be due to solar-type stars, and indeed some solartype exoplanet hosts have already been identified (e.g., [125]). Finally, microlensing surveys are most sensitive to planets with a projected separation from their parent star of roughly twice the snow line. See [43] for a review of microlensing searches for planets. The results from the MOA-II analysis provided strong evidence for a break in the mass ratio function (the distribution of mass ratios) at a mass ratio of $q_{\mathrm{br}}=1.7 \times 10^{-4}$, roughly that of Neptune and the Sun (hereafter NSMR). Parameterizing the mass ratio $q$ and projected separation distribution, $s$, as a broken power law, they find:

$$
\frac{d N}{d \log q d \log s}=0.61_{-0.16}^{+0.21}\left[\left(\frac{q}{q_{\mathrm{br}}}\right)^{-0.93 \pm 0.13} \Theta\left(q-q_{\mathrm{br}}\right)+\left(\frac{q}{q_{\mathrm{br}}}\right)^{0.6_{-0.4}^{+0.5}} \Theta\left(q_{\mathrm{br}}-q\right)\right] s^{0.49_{-0.49}^{+0.47}},
$$

where $\Theta(x)$ is the Heaviside step function. This implies that the mass ratio function of cold exoplanets with mass ratios above that of the NSMR is steeper than by found by [28]. In addition, [120] found that the distribution of planets beyond the snow line is consistent with a log-uniform (Öpik [97]) distribution. Finally, the results of 120 imply that NSMR planets are likely the most common planets beyond the snow line. We note that these results were subsequently refined by [123] and 61].

\footnotetext{
${ }^{5}$ A directly-imaged planetary mass companion was discovered around the brown dwarf primary, 2MASS 1207 24], in 2005, but this system likely formed in manner more akin to a stellar binary, highlighting the importance of mass ratio in assessing the planetary nature of low mass companions.

${ }^{6}$ Although, we note that it is possible to estimate the host star mass and thus the planet mass using a variety of techniques (see 6]), and for the forthcoming survey by the Nancy Grace Roman Space telescope, such measurements should be routine [7.
} 




Figure 2: Modified from Fig. 14 of Kunimoto \& Matthews (2020), showing a collection of $\Gamma_{\oplus}$ values from the literature: 69, 98, 55, 18, 140, 41, 64, 92, 19, 37, 101, 31, 137. Squares indicate that grid-based occurrence rates were explored [69, 55, 101, while circles indicate a functional form was fitted and integrated for the occurrence rate (all others).

The transit detection technique allows for a much larger number of stars to be surveyed at once, albeit with strict orientation requirements, and at greater orbital separations for low-mass planets, than the radial velocity technique (although still smaller orbital separations than are probed by microlensing). Some early transit demographics studies took advantage of this to examine nearby star clusters as laboratories for measuring occurrence rates as a function of controlled variables, e.g. metallicity and stellar density in 47 Tuc, $\omega$ Centauri, and other clusters [90, 20, 45, 133, 132. However, the first large-scale statistical studies with transiting exoplanets were performed with data from the NASA Kepler mission [14. Prior to its launch in 2009 there were $\sim 50$ known transiting planets; to date an additional $\sim 2400$ planets have come from its prime mission dataset, ushering in the statistical age of exoplanets.

Some important results from Kepler include the bi-modality of the size distribution of small planets [40, the discovery of the hot Neptune desert [86, the clustering of planet sizes within planetary systems 130, 51, and the decreasing metallicity dependence of planet occurrence rate with decreasing planet size [102. The primary goal of the mission was to measure $\eta_{\oplus}$, however a combination of the unexpectedly high noise properties of sun-like stars and several hardware issues led to a final dataset that is incomplete in the relevant parameter space. Nevertheless, there have been many increasingly sophisticated attempts to constrain $\eta_{\oplus}$. These are summarized in Figure 2 via a metric labelled $\Gamma_{\oplus}$, which is the value of the occurrence rate model (whether a functional fit in radius/period space or a grid of discrete bins over radius and period) evaluated around the values for Earth $\left(P=365.25 \mathrm{~d}, R_{p}=1.0 R_{\oplus}\right)$. The most recent investigations from 2019 and 2020 include robust corrections for completeness and reliability, and vary mostly in the functional form they fit to the underlying population, and the parameter space over which it is fit. From a spread of over three orders of magnitude in the first few years of analysis, the community now appears to be converging on an $\eta_{\oplus}$ value between 5-50\%, with the remaining uncertainly arising largely from the small number of detections in the relevant parameter space.

Direct imaging surveys, enabled by state-of-the-art extreme (defined by actuator density on deformable 
mirrors) adaptive optics (AO) on 6-12 meter telescopes, are limited to gas giants $>1$ Jupiter mass beyond 10 AU found around nearby young stars, as well as brown dwarf companions that could be a natural extension of the stellar companion mass ratio distribution (e.g., [111). Early studies made predictions of expected yields based on extrapolations of the power-law models from [28] and fitted null results with an outer cutoff radius $(\mathbf{7 0}, 94,52,127)$. Such studies are done in the wavelength range from 1-4 microns as young planets $(<300 \mathrm{Myr})$ are brighter and hotter than their older counterparts, and current AO systems perform well ([77, 9, 49). It can be shown that the L-band (3.6-4.0 microns) is preferred for slightly older stars which enable cohorts of closer stars to comprise target samples [52]. The youngest stars are rare in the solar neighborhood, therefore target stars are generally $15-150$ pc away. Results to date can be summarized as: a) gas giant planets beyond 10-50 $\mathrm{AU}$ are rare; b) wide orbit gas giants from 1-15 Jupiter mass appear to be more common around higher mass stars, but are also consistent with a common mass ratio distribution 128]; c) brown dwarf companions (20-76 Jupiter masses) are more common around M dwarfs compared to higher mass stars [95, though consistent with a common companion mass ratio distribution [128]; and d) the eccentricity distribution may present a dichotomy where lower planetary mass companions have lower eccentricities compared to brown dwarf companions [17. Pioneering attempts are just being made to image planets that are in thermal equilibrium with the closest stars to the Sun (e.g. $\epsilon$ Eri b discovered by RV with imaging attempts by [83] and references therein). With temperatures of 100-500 K, set by stellar luminosity and orbital radius, observations are made from 5-13 microns around nearby stars with spectral type $>$ K5 . However, even detecting a super-Earth around Alpha Cen A at $1.3 \mathrm{pc}$ would take many nights of observtions (c.f. 62]).

\section{Important questions and goals}

As is clear from Figure 1 our census of exoplanets remains substantially incomplete, and as discussed below, practical obstacles have hindered attempts to combine statistical constraints from multiple surveys and detection methods. Many major questions about the demographics of exoplanets remain. We mention a few of the most notable here, but emphasize that this list is not meant to be complete.

- How common are solar system analogs? One of the most surprising results from Kepler is that the majority of stars appear to host relatively close-in, compact systems of super-Earths and/or subNeptunes (e.g., [53, 137, 31, 121). As our solar system does not host any analogues of such planets; this suggests that planetary architectures like our own (with small rocky planets in the temperate zone and gas giants beyond the ice line) may not be common. However, given that most detection methods are not yet sensitive to analogs of the majority of the planets in our solar system, it is still not clear how rare planetary systems like ours are. Even if gas giants beyond the ice line are realized in $10 \%$ of systems, and the frequency of habitable zone terrestrial planets is $10 \%$, these properties could be correlated such that the joint probability could be $>1 \%$.

- How does the shape of the exoplanet mass function depend on semimajor axis and host star mass? The exoplanet mass function (or mass ratio function) has been measured over many orders of magnitude using RV and microlensing surveys. The radius distribution has been measured by Kepler, which can be converted to mass function by adopting a mass-radius relation (e.g., [25]). Generally, the mass function measured by these surveys over their full range is known to be inconsistent with a single power law (e.g., [120, 98,). However, it is unclear if and how the normalization and detailed shape of the mass function (including any slopes and breaks of a piecewise power-law fit) depend on semimajor axis (particularly on either side of the snow line) and/or host star mass.

- Which is the more fundamental parameter that determines the final architectures of planetary systems: mass or mass ratio? The physical mechanisms that govern planet formation and evolution can depend more directly on either the planet mass or planet/star mass ratio. For example, the critical mass for nucleated runaway gas accretion is more directly dependent on the core mass [89, 104, 107, and thus (along with the disk lifetime) may influence the frequency of "failed Jupiters". On the other hand, the final mass of a giant planet formed by core accretion likely depends on many factors, including the the Hill radius of an exoplanet, as well as the properties and lifetime 
of the disk [114. In the traditional model of solid accretion, the "isolation mass" depends on the mass ratio (via the Hill radius). However, when accounting for migration and/or pebble accretion, the importance of the isolation mass is less clear (see [115] and references therein). Thus determining whether mass or mass ratio is the primary physical parameter that governs planet demographics in various regimes may help elucidate the physical of planet formation at various stages. We note that there is preliminary evidence that the mass ratio function may be more universal [98].

- How do the detailed architectures of planetary systems depend on the properties and environment of the host star? There is substantial evidence that the frequency of relatively short-period giant planets depends on the mass and metallicity $(\mathrm{Fe} / \mathrm{H})$ of the host star (e.g., [46, 36, [57]). However, the precise dependence of the frequency of less-massive short-period planets on these quantities is less clear and likely more complex (e.g., [53, 32, 38, 102, 139, 72]). For wide-separation giant planets detected by direct imaging, there is some evidence for a dependence of the occurrence rate on spectral type, where higher mass stars are more likely to have gas giant companions [128. In the most general terms, setting aside zones of dynamical instability, the bulk frequency of planets does not appear to depend strongly on stellar multiplicity, although significant work is needed to determine the effects of stellar companions (mass, semimajor axis, and eccentricity) on the detailed architectures of planetary systems (see the discussion in [11] and references therein). The demographics of planetary systems as a function of stellar age has barely been explored (see [8] and references therein), primarily because of the notorious difficultly of determining precise ages of isolated field stars, particularly in the pre-Gaia era. Finally, there have been relatively few explorations of the effect of detailed abundances (e.g., $[\alpha / \mathrm{Fe}]$ ) on exoplanet demographics, primarily because the host stars for the largest statistical sample of planets available, i.e., Kepler, are generally too faint for detailed abundances determinations. One well-posed question that has not yet been unambiguously answered is whether there is a minimum metallicity below which planet formation is inefficient or impossible [45, 118, 33.

- Are there multiple formation channels for giant exoplanets? The two most common models for the giant planet formation 7 are the bottom-up core accretion or nucleated instability model [104, and the top-down gravitational instability model [15. Several theoretical studies have argued that the latter mechanism is only likely to operate in the outer parts of massive disks, and typically forms fairly massive giant planets [106, 68. A related question is whether or not the wide-separation giant planets that have been detected by direct imaging surveys are a distinct population of objects, or are simply the long-period, high-mass tail of planets indirectly detected by RV surveys [16], or the low-mass tail of the stellar companion mass ratio distribution (c.f. [127, 128]). There is suggestive evidence based on segregation in the mass functions, metallicity, and orbital eccentricity distributions of giant planets that at least two formation mechanisms may operate [116, 17.

- How many planets become unbound from their host stars, and what is the mass function of these "free floating" planets? The detection of the first Hot Jupiters provided evidence of large-scale migration of giant planets [74, 108, with several models to explain this migration invoking dynamical instabilities. These would not only form Hot Jupiters, but would also lead to the ejection of protoplanets or planets. Classical models of planet formation include competition and interaction of multiple planetary embryos $\left(5-15 M_{\oplus}\right)$, some of which get ejected (e.g., 4]). Indeed, it has since been proposed that our own solar system may have experienced a much less quiescent formation history than has previously been assumed; such a formation history would also lead to the ejection of a significant number of planetary-mass bodies (see [110] for a review). In addition, other mechanisms including star cluster dynamics have been proposed that can unbind planets from their host stars [1, 126]. Therefore, in principle, the abundance and mass function of free-floating planetary-mass objects can provide a constraint on models of the formation and evolution of planetary systems (as well as the low mass end of the initial mass function of brown dwarfs).

\footnotetext{
${ }^{7}$ We will assume that low-mass planets, and in particular rocky planets, must primarily be formed in a bottom-up scenario.
} 


\section{Challenges}

There are many challenges to determining the demographics of exoplanets over a broad region of parameter space. These challenges have been identified by several authors, and methods to mitigate these challenges have also been identified. Here we will simply provide a list of these challenges, and highlight a few studies that have attempted to address them.

- Intrinsic biases and sensitivities of different exoplanet detection methods. The primary exoplanet detection methods at our disposal (RV, transits, microlensing, direct imaging, and astrometry) are all endowed with their unique biases and planet detection sensitivities. Fortunately, these methods are largely complementary. As a result, by properly combining the results from diverse surveys, it is possible to derive constraints on nearly the entire region of parameter space. However, combining the results of different surveys using the same detection method, let alone combining the results of surveys based on different detection methods, is not trivial. In particular, it is crucial that both the completeness and reliability of individual surveys be carefully quantified. Furthermore, given that different methods are sensitive to different planetary properties, there are often auxiliary constraints or priors that must be adopted and/or marginalized over to combine results from different methods. As a specific example, combining the results from transit surveys and radial velocity surveys requires adopting a mass-radius relation [25, 113. An additional complication is that mass-radius relationships for exoplanets are not completely deterministic, and thus the dispersion in these relations must also be considered [134]. Likely due to these challenges, there have been few attempts to combine demographic constraints from multiple detection methods, with some notable exceptions ([53, 26, 27, 88]).

- Comparing constraints from microlensing surveys to those from other techniques.. Microlensing is relatively unique amongst exoplanet detection methods in that (in general) the host star mass and distance is not constrained by the routine observables (specifically the microlensing light curve). Generally, the only direct observables are the planet/host mass ratio and the projected separation of the planet in units of the angular Einstein ring of the host. However, for planetary microlensing events, it is typically possible to estimate the angular Einstein ring radius. Furthermore, with high angular resolution observations with large telescopes with adaptive optics (e.g., Keck or Very Large Telescope), it is possible to measure the flux from the host star lens (e.g., [10]). Combining this with the measurement of the angular Einstein ring radius, it is possible to estimate the mass and distance to the lens, and the mass of the planet and projected separation of the planet in physical units. With the Nancy Grace Roman Space Telescope (née WFIRST) Galactic Exoplanet survey [119, it will be possible to estimate the mass and distance to the primary lens, and thus the mass of the planet, for the majority of the detected planets [6]. This will allow the direct comparison of microlensing demographics constraints to those from other techniques, and in particular the dependence of exoplanet demographics on host star mass.

- Determining the demographics of exoplanets as a function of the properties and environmental conditions of the host star. It is observationally expensive to precisely constrain the parameters (e.g., mass, radius, abundance, age) and environment (e.g. multiplicity), of exoplanet host stars, particularly for relatively faint hosts. In some cases the multiplicity itself has an impact on the quality of the data one is able to obtain (e.g RV and direct imaging). The largest statistical survey undertaken, i.e. Kepler, targeted a relatively narrow and relatively faint population of main-sequence FGK stars, whose parameters were initially derived using only broad and narrow band photometry [5. These photometrically-derived parameters turned out to be significantly biased, particularly for the cooler stars [80]. Radial velocity surveys typically target an even narrower range of quiet FGK main-sequence stars that have been screened for low stellar activity and relatively slow rotation rates. Larger surveys like K2 and TESS will greatly expand the types of stars that are targeted for exoplanet searches, but the problem of accurately (and uniformly) deriving their stellar parameters remains. Several large-scale spectroscopic surveys (e.g. LAMOST [76], APOGEE [2], GALAH [30]) are publicly releasing hundreds of thousands to millions of stellar spectra. In combination with the precise distances from the ESA Gaia mission, careful treatment of these spectra, including calibration across surveys to remove systematic offsets, and cross-matching with target lists of K2 and TESS, will enable us to 
constrain exoplanet demographics much more robustly as a function of stellar parameters in the near future.

- Determining the effect of exoplanet multiplicity on their demographics. All of the methods that have been used to detect exoplanets (pulsar timing, radial velocities, transits, microlensing, and direct imaging) have detected multi-planet systems, and there is little doubt that astrometry will also detect multi-planet systems as well; multi-planet systems therefore appear to be common. Indeed, it is not always widely appreciated that one of the most fundamental questions one can ask about exoplanet demographics, namely "How common are exoplanets?", is not well-posed. This question could be rephrased as either "What fraction of stars host (at least one) exoplanet?", or "What is the average number of exoplanet per star?". These are two different questions with two distinct answers. For instance, there are a number of lines of evidence from Kepler that multi-planet systems may be distinct from systems with only one detected planet 75, 56, 3, 140. In addition, there is evidence that Kepler's multi-planet systems tend to be "peas in a pod", e.g., that the planets in such systems tend to have similar radii and are regularly-spaced [130, or to be clustered in size and spacing [51] (although c.f. [93, 138, 131]). Accounting for the effect of planetary system multiplicity may change $\eta_{\oplus}$ by an amount that is larger than the currently stated uncertainties on that value (see, e.g. [140]).

- Calibrating the mass-age-luminosity relationship of young exoplanets. Observations of young, self-luminous, directly-detected planets can provide estimates of their luminosity, and temperature, derived from spectral energy distributions as well as spectroscopy, the combination of which provides a coarse radius estimate. While some spectral features are known to be sensitive to surface gravity, inferring the mass of such directly-imaged planets requires an estimate of the age of the system, as such planets gradually cool as they radiate residual energy from formation 21. With an age and luminosity, it is possible to use models to infer the mass of the planet, modulo an assumption of the specific entropy of the forming planet. In principle, there is a unique temperature associated with a luminosity and age as a function of specific entropy. However, these models are only now being calibrated with direct imaging observations of objects with dynamical mass estimates and known age (e.g. [117; c.f. section 3 of [16]). Legacy radial velocity surveys as well as Gaia provide opportunities to rectify the situation.

- Direct Detection of Earth Analogs. Mature planets that are in thermal equilibrium with their host star have two primary components to their spectrum: the reflected light and the thermal emission from reprocessed stellar radiation. The amount of reflected light depends on the product of the radius and the albedo, modified by the (wavelength-dependent) scattering phase function. The thermal emission spectrum of such planets depends on the temperature of the planet (which in turn depends on the scattering albedo). Thus, by measuring both the reflected light and thermal emission of planets in equilibrium, it is possible to infer the radius, albedo, and temperature of mature directly-imaged planets. Unfortunately, directly detecting mature planets in either reflected light or thermal emission from the ground will require future Extremely Large Telescopes (ELTs) with adaptive optics, and even then will only be possible for a relatively small sample of the most nearby targets. Furthermore, the best targets for reflected light observations are planets orbiting low-mass stars, whereas the best targets for thermal emission are planets orbiting FGK stars. Nevertheless, it may be possible to detect both signals for a handful of planets with radius $>2 R_{\oplus}$ orbiting inside the habitable zones of the most nearby stars (should the planets exist) [129]. True Earth analogs orbiting in the habitable zones of FGK stars may be out of reach of ground-based facilities. Detection of significant samples of such planets will require specially-designed space-based missions operating in the UV/optical/near-infrared such as the Habitable Exoplanet Observatory (HabEx, [4]), or the Large UV/Optical/InfraRed Mission (LUVOIR, 122]), or space-based missions operating in the mid-infrared $(\sim 10 \mu \mathrm{m})$ such as the Large Interferometer for Exoplanets (LIFE, [105]) (thermal emission). We also note that direct detection of true Earth analogues (around Sun-like stars) via secondary eclipse is well beyond the capabilities of JWST.

- Measuring the masses of potentially habitable directly-imaged planets orbiting nearby solar-type stars. There are several proposed mission concepts that have as one of their primary goals to directly detect and take the spectrum of terrestrial planets in the habitable zones of nearby 

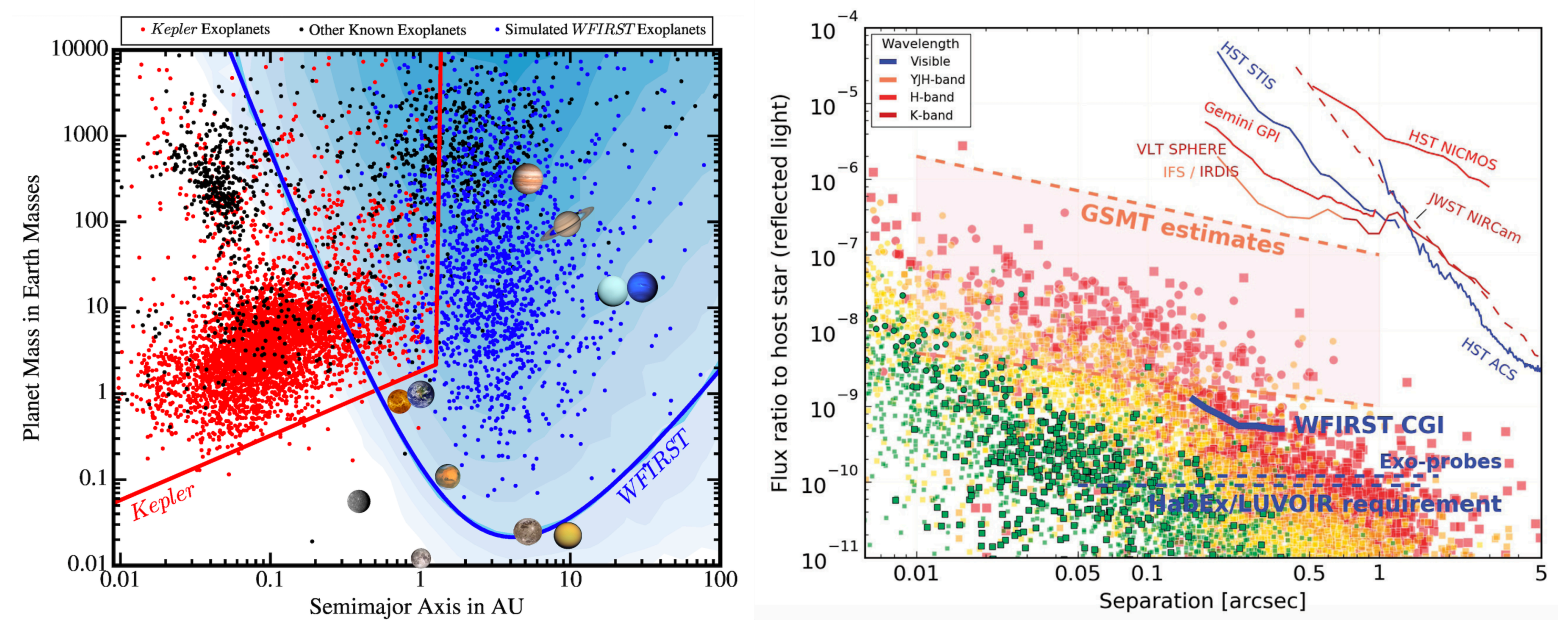

Figure 3: (left) The region of sensitivity of the Kepler prime mission (red solid lines) versus the predicted region of sensitivity of the Roman (née WFIRST) Galactic Exoplanet Survey (blue solid lines). From 99. Courtesy of M. Penny. (C) AAS. Reproduced with permission. (right) Reflected light flux ratio versus angular separation for current and future direct imaging surveys. The markers denote simulated planet populations within $27 \mathrm{pc}$ using occurrence rates from 65. Various cuts have been applied to more realistically portray the detectability of these populations. The marker size is proportional to planet size and colors denote specific bins: giant planets (red); Neptunes (orange), mini-Neptunes (yellow); super-Earths (dark green); temperate Earths and super-Earths (light green). Round points indicate planets orbiting cool stars $\left(T_{\text {eff }}<4000 \mathrm{~K}\right)$, while square points denote planets around warmer stars. From Fig. 4.3 in [96]; see that reference for additional details. Republished with permission of the National Academies Press; permission conveyed through Copyright Clearance Center, Inc. Courtesy of D. Mawet, B. Macintosh, T. Meshkat, V. Bailey, and D. Savransky.

sunlike stars (e.g., HabEx, LUVOIR, and LIFE). The interpretation of the spectra of such planets obtained by these missions would be greatly simplified if the masses of these planets were known. Unfortunately, the RV signal of an Earth-Sun analog is only $\sim 9 \mathrm{~cm} / \mathrm{s}$, which is a factor of $4-5$ smaller than the current state-of-the-art for ground-based RV surveys. As a result, substantial effort and investments have been put into improving the precision and accuracy of RV surveys to $\sim 10 \mathrm{~cm} / \mathrm{s}$ and below [35, 103. The astrometric signal of an Earth-Sun analog is only $\sim 0.3 \mu$ as, which is almost certainly not achievable from the ground. Several mission concepts studies that may enable astrometric precisions well below these levels have been proposed (e.g., [78).

\section{Opportunities}

In this section, we briefly summarize some of the future opportunities for refining and expanding the demographics of exoplanets. In particular, we will focus on the prospects for exploring the region of planet parameter space (planet mass, radius, semimajor axis, and period) for which exoplanet demographics can be determined, as well as the region of host star parameter space (mass, temperature, abundance, multiplicity, and age). As we are focusing on demographics, we will not discuss the many exciting future prospects for detailed atmospheric characterization of exoplanets using, e.g., current and future ground-based facilities (including ELTs), current and future space-based facilities such as the Hubble Space Telescope (HST), the CHaracterising ExOPlanet Satellite (CHEOPS), the James Webb Space Telescope (JWST), Twinkle, the Nancy Grace Roman Space Telescope (Roman), and the Atmospheric Remote-sensing Infrared Exoplanet Large-survey (ARIEL), or proposed missions such as Origins.

- The Transiting Exoplanet Survey Satellite (TESS): The NASA TESS mission 112 will observe almost the entire sky at 30-minute cadence with observing baselines of 27-351 d; transiting exoplanet 
detections are expected down to $T_{\mathrm{mag}}=14$. While the range in planet radius and period covered by TESS is generally narrower than that covered by Kepler, the target list is several orders of magnitude larger. Thus, although the primary goal of TESS is to provide a sample of small $\left(<4 R_{\oplus}\right)$ transiting planets orbiting bright stars, it will nevertheless contribute significantly to our understanding of exoplanet demographics. In particular, the TESS target sample includes stars covering a much larger range of stellar properties. This fact, combined with the fact that the host stars will be relatively bright and thus amenable to detailed characterization, will enable more in-depth investigations of planet demographics as a function of, e.g., stellar age, mass, temperature, and metallicity. This information will provide important constraints on the dominant physical processes sculpting the planet distributions we see. In addition, the large sample of planets with well-constrained masses and radii amenable to atmospheric characterization will allow us to expand our demographic studies to include atmospheric properties.

- Gaia: ESA's Gaia mission possesses revolutionary capabilities to discover planets down to Neptune masses around nearby low mass stars at relatively large orbital radii via astrometry. Previous estimates that extrapolate radial velocity distributions 28 predict Gaia will discover thousands of planets below a few Jupiter masses (e.g. [100]). However, if the orbital radius distributions peak at $<10$ AU these estimates are likely too optimistic. Nonetheless, Gaia will provide dozens to hundreds of planets with dynamical masses and full Keplerian orbital elements (up to a two-fold degeneracy). These systems can be used to explore the architectures and (in particular) the coplanarity of planetary systems. Furthermore, many of these systems will be amenable to characterization by direct imaging, offering unprecedented opportunities to compare the properties of wide-orbit planets with close-in planets discovered via transit.

- JWST: In the background limit, JWST's ability to directly detect exoplanets will outperform groundbased telescopes by orders of magnitude in the infrared, enabling the discovery of planets $>10$ Earth masses around nearby (10-50 pc), young (30-300 Myr), late-type stars (> M0). However, in the contrast-limit, it is likely that ground-based telescopes with extreme AO will outperform JWST within $\sim 10 \lambda /$ D. Spectral retrieval studies will benefit tremendously from the higher signal-to-noise ratio and wavelength coverage of JWST relative to ground-based telescopes, providing constraints on volatile composition to confront planet formation theory.

- Extremely Large Telescopes (ELTs): ELTs will provide the angular resolution and sensitivity to detect planets below 1 Jupiter mass around nearby stars, many of which will have dynamical masses from radial velocity measurements or Gaia. This will be the first opportunity to characterize a large sample of mature wide-orbit planets to compare with gas giants found around young stars. ELTs will enable imaging at angular scales around stars close enough that imaging and radial velocity (and astrometric) samples will have large overlap in orbital radius, on both sides of the local maxima between 1-10 AU. In addition, ELTs will be able to directly detect planets down to $<2$ Earth radii around the very nearest stars, including possibly in the habitable zone. Thermal infrared imaging will prefer stars earlier than K5 while reflected light studies will be best around M dwarfs, particularly as AO performance pushes into the visible (e.g., [79]).

- The Nancy Grace Roman Space Telescope (Roman): Roman will perform the Roman Galactic Exoplanet Survey (RGES) using the microlensing detection method. Initial estimates from the RGES survey predict that it will detect $\sim 1500$ cold planets (planets beyond the snow line), with masses down to the mass of Ganymede [99. When combined with Kepler, Roman will complete the statistical census of planets with masses and radii greater than roughly that of the Earth, for semimajor axes from zero to infinity; see Figure 3. Indeed, Roman will be sensitive to free-floating planets (planets that are not bound to any star) with masses down to the masses of Mars [59. Together, Kepler and Roman will provide the empirical ground truth that any ab initio planet formation theories must match. Roman will also be able to constrain the frequency of potentially habitable planets orbiting sunlike stars $\left(\eta_{\oplus}\right)$, complementing and bolstering the estimates from Kepler.

- The PLAnetary Transits and Oscillations of stars (PLATO) mission: PLATO [109] is a selected medium-class ESA mission whose objective is to discover and characterize a large number of 
transiting exoplanets, as well as perform asterosiesmology on a large number of stars, including the host stars of a significant fraction of the detected transiting planets. PLATO can be thought of as a next-generation Kepler-like survey: in particular, it will improve upon the primary Kepler survey by observing a larger area of the sky. Therefore, PLATO's target stars will typically be brighter than that of Kepler, thereby facilitating follow-up of the planetary systems, including the ability to measure masses of detected transiting planets with radial velocities. Notably, PLATO will enable a detailed exploration of planet demographics as a function of the stellar age, accessible with asteroseismology, and may also provide an improved estimate of $\eta_{\oplus}$.

- Space-based direct imaging surveys: Future proposed direct-imaging surveys, such as HabEx 44, LUVOIR [122], and LIFE [105] will enable a direct census of nearby $(d \leq 10 \mathrm{pc})$ planetary systems over nearly the entire range of semimajor axes spanned by the planets in our solar system (see Fig. 3 p for the parameter space to which HabEx and LUVOIR are expected to be sensitive). In particular, these missions would enable the detection, as well as orbital and atmospheric characterization, of analogs of our own Venus, Earth, and Jupiter. Depending on the precise architecture and starlight suppression technique, these missions may also be able to detect, and potentially characterize, analogs to Mars and Saturn. Depending on the contrast floor and outer working angle, they may also be able to detect and possibly characterize gas giants at separations corresponding to the ice giants in our solar system (see, e.g., [44]). In other words, these missions have the potential to detect and characterize analogs to nearly all of the planets in our solar system, in individual systems. As a result, these missions will enable us to ask such fundamental questions as: "What is the conditional probability that, given the existence of a terrestrial world in the habitable zone of a nearby star, that the system hosts a giant planet beyond the snow line?"

\section{Epilogue}

The future of exoplanet demographics looks bright. Of the three pillars of the study of exoplanets outlined in the introduction of this chapter, exoplanet demographics is likely the first to reach fruition. This is as it should be. The other two pillars of exoplanet studies: detailed characterization of exoplanets, and the search for habitable worlds and life, fundamentally rely on our basic knowledge of the architectures of planetary systems, and how these architectures depend on the host star properties.

\section{Acknowledgements}

We would like to thank Radek Poleski for assistance with improving Figure 1 Partial support for B.S.G. was provided by the Thomas Jefferson Chair for Space Exploration endowment at the Ohio State University. This research has made use of the NASA Exoplanet Archive, which is operated by the California Institute of Technology, under contract with the National Aeronautics and Space Administration under the Exoplanet Exploration Program.

\section{References}

[1] Fred C. Adams, Eva M. Proszkow, Marco Fatuzzo, and Philip C. Myers. Early Evolution of Stellar Groups and Clusters: Environmental Effects on Forming Planetary Systems. Astrophysical Journal, 641(1):504-525, April 2006.

[2] Shadab Alam, Franco D. Albareti, Carlos Allende Prieto, F. Anders, Scott F. Anderson, Timothy Anderton, Brett H. Andrews, Eric Armengaud, Éric Aubourg, Stephen Bailey, Sarbani Basu, Julian E. Bautista, Rachael L. Beaton, Timothy C. Beers, Chad F. Bender, Andreas A. Berlind, Florian Beutler, Vaishali Bhardwaj, Jonathan C. Bird, Dmitry Bizyaev, Cullen H. Blake, Michael R. Blanton, Michael Blomqvist, John J. Bochanski, Adam S. Bolton, Jo Bovy, A. Shelden Bradley, W. N. Brandt, D. E. Brauer, J. Brinkmann, Peter J. Brown, Joel R. Brownstein, Angela Burden, Etienne 
Burtin, Nicolás G. Busca, Zheng Cai, Diego Capozzi, Aurelio Carnero Rosell, Michael A. Carr, Ricardo Carrera, K. C. Chambers, William James Chaplin, Yen-Chi Chen, Cristina Chiappini, S. Drew Chojnowski, Chia-Hsun Chuang, Nicolas Clerc, Johan Comparat, Kevin Covey, Rupert A. C. Croft, Antonio J. Cuesta, Katia Cunha, Luiz N. da Costa, Nicola Da Rio, James R. A. Davenport, Kyle S. Dawson, Nathan De Lee, Timothée Delubac, Rohit Deshpande, Saurav Dhital, Letícia Dutra-Ferreira, Tom Dwelly, Anne Ealet, Garrett L. Ebelke, Edward M. Edmondson, Daniel J. Eisenstein, Tristan Ellsworth, Yvonne Elsworth, Courtney R. Epstein, Michael Eracleous, Stephanie Escoffier, Massimiliano Esposito, Michael L. Evans, Xiaohui Fan, Emma Fernández-Alvar, Diane Feuillet, Nurten Filiz Ak, Hayley Finley, Alexis Finoguenov, Kevin Flaherty, Scott W. Fleming, Andreu Font-Ribera, Jonathan Foster, Peter M. Frinchaboy, J. G. Galbraith-Frew, Rafael A. García, D. A. García-Hernández, Ana E. García Pérez, Patrick Gaulme, Jian Ge, R. Génova-Santos, A. Georgakakis, Luan Ghezzi, Bruce A. Gillespie, Léo Girardi, Daniel Goddard, Satya Gontcho A. Gontcho, Jonay I. González Hernández, Eva K. Grebel, Paul J. Green, Jan Niklas Grieb, Nolan Grieves, James E. Gunn, Hong Guo, Paul Harding, Sten Hasselquist, Suzanne L. Hawley, Michael Hayden, Fred R. Hearty, Saskia Hekker, Shirley Ho, David W. Hogg, Kelly Holley-Bockelmann, Jon A. Holtzman, Klaus Honscheid, Daniel Huber, Joseph Huehnerhoff, Inese I. Ivans, Linhua Jiang, Jennifer A. Johnson, Karen Kinemuchi, David Kirkby, Francisco Kitaura, Mark A. Klaene, Gillian R. Knapp, Jean-Paul Kneib, Xavier P. Koenig, Charles R. Lam, Ting-Wen Lan, Dustin Lang, Pierre Laurent, Jean-Marc Le Goff, Alexie Leauthaud, Khee-Gan Lee, Young Sun Lee, Timothy C. Licquia, Jian Liu, Daniel C. Long, Martín López-Corredoira, Diego Lorenzo-Oliveira, Sara Lucatello, Britt Lundgren, Robert H. Lupton, III Mack, Claude E., Suvrath Mahadevan, Marcio A. G. Maia, Steven R. Majewski, Elena Malanushenko, Viktor Malanushenko, A. Manchado, Marc Manera, Qingqing Mao, Claudia Maraston, Robert C. Marchwinski, Daniel Margala, Sarah L. Martell, Marie Martig, Karen L. Masters, Savita Mathur, Cameron K. McBride, Peregrine M. McGehee, Ian D. McGreer, Richard G. McMahon, Brice Ménard, Marie-Luise Menzel, Andrea Merloni, Szabolcs Mészáros, Adam A. Miller, Jordi Miralda-Escudé, Hironao Miyatake, Antonio D. Montero-Dorta, Surhud More, Eric Morganson, Xan Morice-Atkinson, Heather L. Morrison, Benôit Mosser, Demitri Muna, Adam D. Myers, Kirpal Nand ra, Jeffrey A. Newman, Mark Neyrinck, Duy Cuong Nguyen, Robert C. Nichol, David L. Nidever, Pasquier Noterdaeme, Sebastián E. Nuza, Julia E. O'Connell, Robert W. O'Connell, Ross O'Connell, Ricardo L. C. Ogando, Matthew D. Olmstead, Audrey E. Oravetz, Daniel J. Oravetz, Keisuke Osumi, Russell Owen, Deborah L. Padgett, Nikhil Padmanabhan, Martin Paegert, Nathalie Palanque-Delabrouille, Kaike Pan, John K. Parejko, Isabelle Pâris, Changbom Park, Petchara Pattarakijwanich, M. PellejeroIbanez, Joshua Pepper, Will J. Percival, Ismael Pérez-Fournon, Ignasi Ṕrez-Ra'fols, Patrick Petitjean, Matthew M. Pieri, Marc H. Pinsonneault, Gustavo F. Porto de Mello, Francisco Prada, Abhishek Prakash, Adrian M. Price-Whelan, Pavlos Protopapas, M. Jordan Raddick, Mubdi Rahman, Beth A. Reid, James Rich, Hans-Walter Rix, Annie C. Robin, Constance M. Rockosi, Thaíse S. Rodrigues, Sergio Rodríguez-Torres, Natalie A. Roe, Ashley J. Ross, Nicholas P. Ross, Graziano Rossi, John J. Ruan, J. A. Rubiño-Martín, Eli S. Rykoff, Salvador Salazar-Albornoz, Mara Salvato, Lado Samushia, Ariel G. Sánchez, Basílio Santiago, Conor Sayres, Ricardo P. Schiavon, David J. Schlegel, Sarah J. Schmidt, Donald P. Schneider, Mathias Schultheis, Axel D. Schwope, C. G. Scóccola, Caroline Scott, Kris Sellgren, Hee-Jong Seo, Aldo Serenelli, Neville Shane, Yue Shen, Matthew Shetrone, Yiping Shu, V. Silva Aguirre, Thirupathi Sivarani, M. F. Skrutskie, Anže Slosar, Verne V. Smith, Flávia Sobreira, Diogo Souto, Keivan G. Stassun, Matthias Steinmetz, Dennis Stello, Michael A. Strauss, Alina Streblyanska, Nao Suzuki, Molly E. C. Swanson, Jonathan C. Tan, Jamie Tayar, Ryan C. Terrien, Aniruddha R. Thakar, Daniel Thomas, Neil Thomas, Benjamin A. Thompson, Jeremy L. Tinker, Rita Tojeiro, Nicholas W. Troup, Mariana Vargas-Magaña, Jose A. Vazquez, Licia Verde, Matteo Viel, Nicole P. Vogt, David A. Wake, Ji Wang, Benjamin A. Weaver, David H. Weinberg, Benjamin J. Weiner, Martin White, John C. Wilson, John P. Wisniewski, W. M. Wood-Vasey, Christophe Ye'che, Donald G. York, Nadia L. Zakamska, O. Zamora, Gail Zasowski, Idit Zehavi, Gong-Bo Zhao, Zheng Zheng, Xu Zhou, Zhimin Zhou, Hu Zou, and Guangtun Zhu. The Eleventh and Twelfth Data Releases of the Sloan Digital Sky Survey: Final Data from SDSS-III. Astrophysical Journal Supplement Series, 219(1):12, July 2015.

[3] Sarah Ballard and John Asher Johnson. The Kepler Dichotomy among the M Dwarfs: Half of Systems 
Contain Five or More Coplanar Planets. Astrophysical Journal, 816(2):66, January 2016.

[4] Thomas Barclay, Elisa V. Quintana, Sean N. Raymond, and Matthew T. Penny. The Demographics of Rocky Free-floating Planets and their Detectability by WFIRST. Astrophysical Journal, 841(2):86, June 2017.

[5] Natalie M. Batalha, William J. Borucki, David G. Koch, Stephen T. Bryson, Michael. R. Haas, Timothy M. Brown, Douglas A. Caldwell, Jennifer R. Hall, Ronald L. Gilliland, David W. Latham, Soren Meibom, and David G. Monet. Selection, Prioritization, and Characteristics of Kepler Target Stars. Astrophysical Journal Letters, 713(2):L109-L114, April 2010.

[6] David P. Bennett, Jay Anderson, and B. Scott Gaudi. Characterization of Gravitational Microlensing Planetary Host Stars. Astrophysical Journal, 660(1):781-790, May 2007.

[7] David P. Bennett and Sun Hong Rhie. Simulation of a Space-based Microlensing Survey for Terrestrial Extrasolar Planets. Astrophysical Journal, 574(2):985-1003, August 2002.

[8] Travis A. Berger, Daniel Huber, Eric Gaidos, Jennifer L. van Saders, and Lauren M. Weiss. The Gaia-Kepler Stellar Properties Catalog. II. Planet Radius Demographics as a Function of Stellar Mass and Age. Astronomical Journal, 160(3):108, September 2020.

[9] J. L. Beuzit, A. Vigan, D. Mouillet, K. Dohlen, R. Gratton, A. Boccaletti, J. F. Sauvage, H. M. Schmid, M. Langlois, C. Petit, A. Baruffolo, M. Feldt, J. Milli, Z. Wahhaj, L. Abe, U. Anselmi, J. Antichi, R. Barette, J. Baudrand, P. Baudoz, A. Bazzon, P. Bernardi, P. Blanchard, R. Brast, P. Bruno, T. Buey, M. Carbillet, M. Carle, E. Cascone, F. Chapron, J. Charton, G. Chauvin, R. Claudi, A. Costille, V. De Caprio, J. de Boer, A. Delboulbé, S. Desidera, C. Dominik, M. Downing, O. Dupuis, C. Fabron, D. Fantinel, G. Farisato, P. Feautrier, E. Fedrigo, T. Fusco, P. Gigan, C. Ginski, J. Girard, E. Giro, D. Gisler, L. Gluck, C. Gry, T. Henning, N. Hubin, E. Hugot, S. Incorvaia, M. Jaquet, M. Kasper, E. Lagadec, A. M. Lagrange, H. Le Coroller, D. Le Mignant, B. Le Ruyet, G. Lessio, J. L. Lizon, M. Llored, L. Lundin, F. Madec, Y. Magnard, M. Marteaud, P. Martinez, D. Maurel, F. Ménard, D. Mesa, O. Möller-Nilsson, T. Moulin, C. Moutou, A. Origné, J. Parisot, A. Pavlov, D. Perret, J. Pragt, P. Puget, P. Rabou, J. Ramos, J. M. Reess, F. Rigal, S. Rochat, R. Roelfsema, G. Rousset, A. Roux, M. Saisse, B. Salasnich, E. Santambrogio, S. Scuderi, D. Segransan, A. Sevin, R. Siebenmorgen, C. Soenke, E. Stadler, M. Suarez, D. Tiphène, M. Turatto, S. Udry, F. Vakili, L. B. F. M. Waters, L. Weber, F. Wildi, G. Zins, and A. Zurlo. SPHERE: the exoplanet imager for the Very Large Telescope. Astronomy \& Astrophysics, 631:A155, November 2019.

[10] A. Bhattacharya, J. P. Beaulieu, D. P. Bennett, J. Anderson, N. Koshimoto, J. R. Lu, V. Batista, J. W. Blackman, I. A. Bond, A. Fukui, C. B. Henderson, Y. Hirao, J. B. Marquette, P. Mroz, C. Ranc, and A. Udalski. WFIRST Exoplanet Mass-measurement Method Finds a Planetary Mass of $39 \pm 8 \mathrm{M}$ $\oplus$ for OGLE-2012-BLG-0950Lb. Astronomical Journal, 156(6):289, December 2018.

[11] Mariangela Bonavita and Silvano Desidera. Frequency of Planets in Binaries. Galaxies, 8(1):16, February 2020.

[12] I. A. Bond, A. Udalski, M. Jaroszyński, N. J. Rattenbury, B. Paczyński, I. Soszyński, L. Wyrzykowski, M. K. Szymański, M. Kubiak, O. Szewczyk, K. Żebruń, G. Pietrzyński, F. Abe, D. P. Bennett, S. Eguchi, Y. Furuta, J. B. Hearnshaw, K. Kamiya, P. M. Kilmartin, Y. Kurata, K. Masuda, Y. Matsubara, Y. Muraki, S. Noda, K. Okajima, T. Sako, T. Sekiguchi, D. J. Sullivan, T. Sumi, P. J. Tristram, T. Yanagisawa, P. C. M. Yock, and OGLE Collaboration. OGLE 2003-BLG-235/MOA 2003-BLG-53: A Planetary Microlensing Event. Astrophysical Journal Letters, 606(2):L155-L158, May 2004.

[13] X. Bonfils, X. Delfosse, S. Udry, T. Forveille, M. Mayor, C. Perrier, F. Bouchy, M. Gillon, C. Lovis, F. Pepe, D. Queloz, N. C. Santos, D. Ségransan, and J. L. Bertaux. The HARPS search for southern extra-solar planets. XXXI. The M-dwarf sample. Astronomy \& Astrophysics, 549:A109, January 2013. 
[14] William J. Borucki, David Koch, Gibor Basri, Natalie Batalha, Timothy Brown, Douglas Caldwell, John Caldwell, Jørgen Christensen-Dalsgaard, William D. Cochran, Edna DeVore, Edward W. Dunham, Andrea K. Dupree, Thomas N. Gautier, John C. Geary, Ronald Gilliland, Alan Gould, Steve B. Howell, Jon M. Jenkins, Yoji Kondo, David W. Latham, Geoffrey W. Marcy, Søren Meibom, Hans Kjeldsen, Jack J. Lissauer, David G. Monet, David Morrison, Dimitar Sasselov, Jill Tarter, Alan Boss, Don Brownlee, Toby Owen, Derek Buzasi, David Charbonneau, Laurance Doyle, Jonathan Fortney, Eric B. Ford, Matthew J. Holman, Sara Seager, Jason H. Steffen, William F. Welsh, Jason Rowe, Howard Anderson, Lars Buchhave, David Ciardi, Lucianne Walkowicz, William Sherry, Elliott Horch, Howard Isaacson, Mark E. Everett, Debra Fischer, Guillermo Torres, John Asher Johnson, Michael Endl, Phillip MacQueen, Stephen T. Bryson, Jessie Dotson, Michael Haas, Jeffrey Kolodziejczak, Jeffrey Van Cleve, Hema Chandrasekaran, Joseph D. Twicken, Elisa V. Quintana, Bruce D. Clarke, Christopher Allen, Jie Li, Haley Wu, Peter Tenenbaum, Ekaterina Verner, Frederick Bruhweiler, Jason Barnes, and Andrej Prsa. Kepler Planet-Detection Mission: Introduction and First Results. Science, 327(5968):977, February 2010.

[15] A. P. Boss. Giant planet formation by gravitational instability. Science, 276:1836-1839, January 1997.

[16] Brendan P. Bowler. Imaging Extrasolar Giant Planets. Proceedings of the Astronomical Society of the Pacific, 128(968):102001, October 2016.

[17] Brendan P. Bowler, Sarah C. Blunt, and Eric L. Nielsen. Population-level Eccentricity Distributions of Imaged Exoplanets and Brown Dwarf Companions: Dynamical Evidence for Distinct Formation Channels. Astronomical Journal, 159(2):63, February 2020.

[18] S. Bryson, J. Coughlin, N. M. Batalha, T. Berger, D. Huber, C. Burke, J. Dotson, and S. E. Mullally. A Probabilistic Approach to Kepler Completeness and Reliability for Exoplanet Occurrence Rates. Astronomical Journal, 159(6):279, June 2020.

[19] Christopher J. Burke, Jessie L. Christiansen, F. Mullally, Shawn Seader, Daniel Huber, Jason F. Rowe, Jeffrey L. Coughlin, Susan E. Thompson, Joseph Catanzarite, Bruce D. Clarke, Timothy D. Morton, Douglas A. Caldwell, Stephen T. Bryson, Michael R. Haas, Natalie M. Batalha, Jon M. Jenkins, Peter Tenenbaum, Joseph D. Twicken, Jie Li, Elisa Quintana, Thomas Barclay, Christopher E. Henze, William J. Borucki, Steve B. Howell, and Martin Still. Terrestrial Planet Occurrence Rates for the Kepler GK Dwarf Sample. Astrophysical Journal, 809(1):8, August 2015.

[20] Christopher J. Burke, B. Scott Gaudi, D. L. DePoy, and Richard W. Pogge. Survey for Transiting Extrasolar Planets in Stellar Systems. III. A Limit on the Fraction of Stars with Planets in the Open Cluster NGC 1245. Astronomical Journal, 132(1):210-230, July 2006.

[21] A. Burrows, M. Marley, W. B. Hubbard, J. I. Lunine, T. Guillot, D. Saumon, R. Freedman, D. Sudarsky, and C. Sharp. A Nongray Theory of Extrasolar Giant Planets and Brown Dwarfs. Astrophysical Journal, 491(2):856-875, December 1997.

[22] Bruce Campbell, G. A. H. Walker, and S. Yang. A Search for Substellar Companions to Solar-type Stars. Astrophysical Journal, 331:902, August 1988.

[23] A. Cassan, D. Kubas, J. P. Beaulieu, M. Dominik, K. Horne, J. Greenhill, J. Wambsganss, J. Menzies, A. Williams, U. G. Jørgensen, A. Udalski, D. P. Bennett, M. D. Albrow, V. Batista, S. Brillant, J. A. R. Caldwell, A. Cole, Ch. Coutures, K. H. Cook, S. Dieters, D. Dominis Prester, J. Donatowicz, P. Fouqué, K. Hill, N. Kains, S. Kane, J. B. Marquette, R. Martin, K. R. Pollard, K. C. Sahu, C. Vinter, D. Warren, B. Watson, M. Zub, T. Sumi, M. K. Szymański, M. Kubiak, R. Poleski, I. Soszynski, K. Ulaczyk, G. Pietrzyński, and Ł. Wyrzykowski. One or more bound planets per Milky Way star from microlensing observations. Nature, 481(7380):167-169, January 2012.

[24] G. Chauvin, A. M. Lagrange, C. Dumas, B. Zuckerman, D. Mouillet, I. Song, J. L. Beuzit, and P. Lowrance. A giant planet candidate near a young brown dwarf. Direct VLT/NACO observations using IR wavefront sensing. Astronomy \& Astrophysics, 425:L29-L32, October 2004. 
[25] Jingjing Chen and David Kipping. Probabilistic Forecasting of the Masses and Radii of Other Worlds. Astrophysical Journal, 834(1):17, January 2017.

[26] Christian Clanton and B. Scott Gaudi. Synthesizing Exoplanet Demographics from Radial Velocity and Microlensing Surveys. II. The Frequency of Planets Orbiting M Dwarfs. Astrophysical Journal, 791(2):91, August 2014.

[27] Christian Clanton and B. Scott Gaudi. Synthesizing Exoplanet Demographics: A Single Population of Long-period Planetary Companions to M Dwarfs Consistent with Microlensing, Radial Velocity, and Direct Imaging Surveys. Astrophysical Journal, 819(2):125, March 2016.

[28] Andrew Cumming, R. Paul Butler, Geoffrey W. Marcy, Steven S. Vogt, Jason T. Wright, and Debra A. Fischer. The Keck Planet Search: Detectability and the Minimum Mass and Orbital Period Distribution of Extrasolar Planets. Proceedings of the Astronomical Society of the Pacific, 120(867):531, May 2008 .

[29] Andrew Cumming, Geoffrey W. Marcy, and R. Paul Butler. The Lick Planet Search: Detectability and Mass Thresholds. Astrophysical Journal, 526(2):890-915, December 1999.

[30] G. M. De Silva, K. C. Freeman, J. Bland-Hawthorn, S. Martell, E. Wylie de Boer, M. Asplund, S. Keller, S. Sharma, D. B. Zucker, T. Zwitter, B. Anguiano, C. Bacigalupo, D. Bayliss, M. A. Beavis, M. Bergemann, S. Campbell, R. Cannon, D. Carollo, L. Casagrande, A. R. Casey, G. Da Costa, V. D'Orazi, A. Dotter, L. Duong, A. Heger, M. J. Ireland, P. R. Kafle, J. Kos, J. Lattanzio, G. F. Lewis, J. Lin, K. Lind, U. Munari, D. M. Nataf, S. O'Toole, Q. Parker, W. Reid, K. J. Schlesinger, A. Sheinis, J. D. Simpson, D. Stello, Y. S. Ting, G. Traven, F. Watson, R. Wittenmyer, D. Yong, and M. Žerjal. The GALAH survey: scientific motivation. Monthly Notices of the Royal Astronomical Society, 449(3):2604-2617, May 2015.

[31] Subo Dong and Zhaohuan Zhu. Fast Rise of "Neptune-size" Planets $\left(4-8 \mathrm{R}_{\oplus}\right)$ from $\mathrm{P} \sim 10$ to $\sim 250$ Days - Statistics of Kepler Planet Candidates up to 0.75 AU. Astrophysical Journal, 778(1):53, November 2013.

[32] Courtney D. Dressing and David Charbonneau. The Occurrence Rate of Small Planets around Small Stars. Astrophysical Journal, 767(1):95, April 2013.

[33] J. P. Faria, N. C. Santos, P. Figueira, A. Mortier, X. Dumusque, I. Boisse, G. Lo Curto, C. Lovis, M. Mayor, C. Melo, F. Pepe, D. Queloz, A. Santerne, D. Ségransan, S. G. Sousa, A. Sozzetti, and S. Udry. The HARPS search for southern extra-solar planets. XL. Searching for Neptunes around metal-poor stars. Astronomy \& Astrophysics, 589:A25, May 2016.

[34] Rachel B. Fernandes, Gijs D. Mulders, Ilaria Pascucci, Christoph Mordasini, and Alexandre Emsenhuber. Hints for a Turnover at the Snow Line in the Giant Planet Occurrence Rate. Astrophysical Journal, 874(1):81, March 2019.

[35] Debra A. Fischer, Guillem Anglada-Escude, Pamela Arriagada, Roman V. Baluev, Jacob L. Bean, Francois Bouchy, Lars A. Buchhave, Thorsten Carroll, Abhijit Chakraborty, Justin R. Crepp, Rebekah I. Dawson, Scott A. Diddams, Xavier Dumusque, Jason D. Eastman, Michael Endl, Pedro Figueira, Eric B. Ford, Daniel Foreman-Mackey, Paul Fournier, Gabor Fürész, B. Scott Gaudi, Philip C. Gregory, Frank Grundahl, Artie P. Hatzes, Guillaume Hébrard, Enrique Herrero, David W. Hogg, Andrew W. Howard, John A. Johnson, Paul Jorden, Colby A. Jurgenson, David W. Latham, Greg Laughlin, Thomas J. Loredo, Christophe Lovis, Suvrath Mahadevan, Tyler M. McCracken, Francesco Pepe, Mario Perez, David F. Phillips, Peter P. Plavchan, Lisa Prato, Andreas Quirrenbach, Ansgar Reiners, Paul Robertson, Nuno C. Santos, David Sawyer, Damien Segransan, Alessandro Sozzetti, Tilo Steinmetz, Andrew Szentgyorgyi, Stéphane Udry, Jeff A. Valenti, Sharon X. Wang, Robert A. Wittenmyer, and Jason T. Wright. State of the Field: Extreme Precision Radial Velocities. Proceedings of the Astronomical Society of the Pacific, 128(964):066001, June 2016. 
[36] Debra A. Fischer and Jeff Valenti. The Planet-Metallicity Correlation. Astrophysical Journal, 622(2):1102-1117, April 2005.

[37] Daniel Foreman-Mackey, David W. Hogg, and Timothy D. Morton. Exoplanet Population Inference and the Abundance of Earth Analogs from Noisy, Incomplete Catalogs. Astrophysical Journal, 795(1):64, November 2014.

[38] François Fressin, Guillermo Torres, David Charbonneau, Stephen T. Bryson, Jessie Christiansen, Courtney D. Dressing, Jon M. Jenkins, Lucianne M. Walkowicz, and Natalie M. Batalha. The False Positive Rate of Kepler and the Occurrence of Planets. Astrophysical Journal, 766(2):81, April 2013.

[39] Benjamin Fulton, Lee Rosenthal, Andrew Howard, Lea Hirsch, and Howard Isaacson. Frequency of Gaseous Planets Beyond the Ice Line. In AAS/Division for Extreme Solar Systems Abstracts, volume 51 of AAS/Division for Extreme Solar Systems Abstracts, page 401.01, August 2019.

[40] Benjamin J. Fulton and Erik A. Petigura. The California-Kepler Survey. VII. Precise Planet Radii Leveraging Gaia DR2 Reveal the Stellar Mass Dependence of the Planet Radius Gap. Astronomical Journal, 156(6):264, December 2018.

[41] Daniel Garrett, Dmitry Savransky, and Rus Belikov. Planet Occurrence Rate Density Models Including Stellar Effective Temperature. Proceedings of the Astronomical Society of the Pacific, 130(993):114403, November 2018.

[42] B. S. Gaudi, M. D. Albrow, J. An, J. P. Beaulieu, J. A. R. Caldwell, D. L. DePoy, M. Dominik, A. Gould, J. Greenhill, K. Hill, S. Kane, R. Martin, J. Menzies, R. M. Naber, J. W. Pel, R. W. Pogge, K. R. Pollard, P. D. Sackett, K. C. Sahu, P. Vermaak, P. M. Vreeswijk, R. Watson, and A. Williams. Microlensing Constraints on the Frequency of Jupiter-Mass Companions: Analysis of 5 Years of PLANET Photometry. Astrophysical Journal, 566(1):463-499, February 2002.

[43] B. Scott Gaudi. Microlensing Surveys for Exoplanets. Annual Reviews of Astronomy and Astrophysics, 50:411-453, September 2012.

[44] B. Scott Gaudi, Sara Seager, Bertrand Mennesson, Alina Kiessling, Keith Warfield, Kerri Cahoy, John T. Clarke, Shawn Domagal-Goldman, Lee Feinberg, Olivier Guyon, Jeremy Kasdin, Dimitri Mawet, Peter Plavchan, Tyler Robinson, Leslie Rogers, Paul Scowen, Rachel Somerville, Karl Stapelfeldt, Christopher Stark, Daniel Stern, Margaret Turnbull, Rashied Amini, Gary Kuan, Stefan Martin, Rhonda Morgan, David Redding, H. Philip Stahl, Ryan Webb, Oscar Alvarez-Salazar, William L. Arnold, Manan Arya, Bala Balasubramanian, Mike Baysinger, Ray Bell, Chris Below, Jonathan Benson, Lindsey Blais, Jeff Booth, Robert Bourgeois, Case Bradford, Alden Brewer, Thomas Brooks, Eric Cady, Mary Caldwell, Rob Calvet, Steven Carr, Derek Chan, Velibor Cormarkovic, Keith Coste, Charlie Cox, Rolf Danner, Jacqueline Davis, Larry Dewell, Lisa Dorsett, Daniel Dunn, Matthew East, Michael Effinger, Ron Eng, Greg Freebury, Jay Garcia, Jonathan Gaskin, Suzan Greene, John Hennessy, Evan Hilgemann, Brad Hood, Wolfgang Holota, Scott Howe, Pei Huang, Tony Hull, Ron Hunt, Kevin Hurd, Sandra Johnson, Andrew Kissil, Brent Knight, Daniel Kolenz, Oliver Kraus, John Krist, Mary Li, Doug Lisman, Milan Mandic, John Mann, Luis Marchen, Colleen Marrese-Reading, Jonathan McCready, Jim McGown, Jessica Missun, Andrew Miyaguchi, Bradley Moore, Bijan Nemati, Shouleh Nikzad, Joel Nissen, Megan Novicki, Todd Perrine, Claudia Pineda, Otto Polanco, Dustin Putnam, Atif Qureshi, Michael Richards, A. J. Eldorado Riggs, Michael Rodgers, Mike Rud, Navtej Saini, Dan Scalisi, Dan Scharf, Kevin Schulz, Gene Serabyn, Norbert Sigrist, Glory Sikkia, Andrew Singleton, Stuart Shaklan, Scott Smith, Bart Southerd, Mark Stahl, John Steeves, Brian Sturges, Chris Sullivan, Hao Tang, Neil Taras, Jonathan Tesch, Melissa Therrell, Howard Tseng, Marty Valente, David Van Buren, Juan Villalvazo, Steve Warwick, David Webb, Thomas Westerhoff, Rush Wofford, Gordon Wu, Jahning Woo, Milana Wood, John Ziemer, Giada Arney, Jay Anderson, Jesús Maíz-Apellániz, James Bartlett, Ruslan Belikov, Eduardo Bendek, Brad Cenko, Ewan Douglas, Shannon Dulz, Chris Evans, Virginie Faramaz, Y. Katherina Feng, Harry Ferguson, Kate Follette, Saavik Ford, Miriam García, Marla Geha, Dawn Gelino, Ylva Götberg, Sergi Hildebrand t, Renyu Hu, Knud Jahnke, Grant Kennedy, Laura Kreidberg, Andrea Isella, Eric Lopez, Franck Marchis, Lucas Macri, Mark Marley, 
William Matzko, Johan Mazoyer, Stephan McCandliss, Tiffany Meshkat, Christoph Mordasini, Patrick Morris, Eric Nielsen, Patrick Newman, Erik Petigura, Marc Postman, Amy Reines, Aki Roberge, Ian Roederer, Garreth Ruane, Edouard Schwieterman, Dan Sirbu, Christopher Spalding, Harry Teplitz, Jason Tumlinson, Neal Turner, Jessica Werk, Aida Wofford, Mark Wyatt, Amber Young, and Rob Zellem. The Habitable Exoplanet Observatory (HabEx) Mission Concept Study Final Report. arXiv e-prints, page arXiv:2001.06683, January 2020.

[45] Ronald L. Gilliland, T. M. Brown, P. Guhathakurta, A. Sarajedini, E. F. Milone, M. D. Albrow, N. R. Baliber, H. Bruntt, A. Burrows, D. Charbonneau, P. Choi, W. D. Cochran, P. D. Edmonds, S. Frandsen, J. H. Howell, D. N. C. Lin, G. W. Marcy, M. Mayor, D. Naef, S. Sigurdsson, C. R. Stagg, D. A. Vandenberg, S. S. Vogt, and M. D. Williams. A Lack of Planets in 47 Tucanae from a Hubble Space Telescope Search. Astrophysical Journal Letters, 545(1):L47-L51, December 2000.

[46] Guillermo Gonzalez. The stellar metallicity-giant planet connection. Monthly Notices of the Royal Astronomical Society, 285(2):403-412, February 1997.

[47] A. Gould, Subo Dong, B. S. Gaudi, A. Udalski, I. A. Bond, J. Greenhill, R. A. Street, M. Dominik, T. Sumi, M. K. Szymański, C. Han, W. Allen, G. Bolt, M. Bos, G. W. Christie, D. L. DePoy, J. Drummond, J. D. Eastman, A. Gal-Yam, D. Higgins, J. Janczak, S. Kaspi, S. Kozłowski, C. U. Lee, F. Mallia, A. Maury, D. Maoz, J. McCormick, L. A. G. Monard, D. Moorhouse, N. Morgan, T. Natusch, E. O. Ofek, B. G. Park, R. W. Pogge, D. Polishook, R. Santallo, A. Shporer, O. Spector, G. Thornley, J. C. Yee, $\mu$ FUN Collaboration, M. Kubiak, G. Pietrzyński, I. Soszyński, O. Szewczyk, Ł. Wyrzykowski, K. Ulaczyk, R. Poleski, OGLE Collaboration, F. Abe, D. P. Bennett, C. S. Botzler, D. Douchin, M. Freeman, A. Fukui, K. Furusawa, J. B. Hearnshaw, S. Hosaka, Y. Itow, K. Kamiya, P. M. Kilmartin, A. Korpela, W. Lin, C. H. Ling, S. Makita, K. Masuda, Y. Matsubara, N. Miyake, Y. Muraki, M. Nagaya, K. Nishimoto, K. Ohnishi, T. Okumura, Y. C. Perrott, L. Philpott, N. Rattenbury, To. Saito, T. Sako, D. J. Sullivan, W. L. Sweatman, P. J. Tristram, E. von Seggern, P. C. M. Yock, MOA Collaboration, M. Albrow, V. Batista, J. P. Beaulieu, S. Brillant, J. Caldwell, J. J. Calitz, A. Cassan, A. Cole, K. Cook, C. Coutures, S. Dieters, D. Dominis Prester, J. Donatowicz, P. Fouqué, K. Hill, M. Hoffman, F. Jablonski, S. R. Kane, N. Kains, D. Kubas, J. B. Marquette, R. Martin, E. Martioli, P. Meintjes, J. Menzies, E. Pedretti, K. Pollard, K. C. Sahu, C. Vinter, J. Wambsganss, R. Watson, A. Williams, M. Zub, PLANET Collaboration, A. Allan, M. F. Bode, D. M. Bramich, M. J. Burgdorf, N. Clay, S. Fraser, E. Hawkins, K. Horne, E. Kerins, T. A. Lister, C. Mottram, E. S. Saunders, C. Snodgrass, I. A. Steele, Y. Tsapras, RoboNet Collaboration, U. G. Jørgensen, T. Anguita, V. Bozza, S. Calchi Novati, K. Harpsøe, T. C. Hinse, M. Hundertmark, P. Kjærgaard, C. Liebig, L. Mancini, G. Masi, M. Mathiasen, S. Rahvar, D. Ricci, G. Scarpetta, J. Southworth, J. Surdej, C. C. Thöne, and MiNDSTEp Consortium. Frequency of Solar-like Systems and of Ice and Gas Giants Beyond the Snow Line from High-magnification Microlensing Events in 2005-2008. Astrophysical Journal, 720(2):1073-1089, September 2010.

[48] A. Gould, S. Dorsher, B. S. Gaudi, and A. Udalski. Frequency of Hot Jupiters and Very Hot Jupiters from the OGLE-III Transit Surveys toward the Galactic Bulge and Carina. Acta Astronomica, 56:1-50, March 2006.

[49] Olivier Guyon. Extreme Adaptive Optics. Annual Reviews of Astronomy and Astrophysics, 56:315355, September 2018.

[50] J. D. Hartman, B. S. Gaudi, M. J. Holman, B. A. McLeod, K. Z. Stanek, J. A. Barranco, M. H. Pinsonneault, S. Meibom, and J. S. Kalirai. Deep MMT Transit Survey of the Open Cluster M37 IV: Limit on the Fraction of Stars with Planets as Small as 0.3R ${ }_{J}$. Astrophysical Journal, 695(1):336-356, April 2009.

[51] Matthias Y. He, Eric B. Ford, and Darin Ragozzine. Architectures of exoplanetary systems - I. A clustered forward model for exoplanetary systems around Kepler's FGK stars. Monthly Notices of the Royal Astronomical Society, 490(4):4575-4605, December 2019. 
[52] A. N. Heinze, Philip M. Hinz, Matthew Kenworthy, Michael Meyer, Suresh Sivanandam, and Douglas Miller. Constraints on Long-period Planets from an L'- and M-band Survey of Nearby Sun-like Stars: Modeling Results. Astrophysical Journal, 714(2):1570-1581, May 2010.

[53] Andrew W. Howard, Geoffrey W. Marcy, Stephen T. Bryson, Jon M. Jenkins, Jason F. Rowe, Natalie M. Batalha, William J. Borucki, David G. Koch, Edward W. Dunham, III Gautier, Thomas N., Jeffrey Van Cleve, William D. Cochran, David W. Latham, Jack J. Lissauer, Guillermo Torres, Timothy M. Brown, Ronald L. Gilliland, Lars A. Buchhave, Douglas A. Caldwell, Jørgen ChristensenDalsgaard, David Ciardi, Francois Fressin, Michael R. Haas, Steve B. Howell, Hans Kjeldsen, Sara Seager, Leslie Rogers, Dimitar D. Sasselov, Jason H. Steffen, Gibor S. Basri, David Charbonneau, Jessie Christiansen, Bruce Clarke, Andrea Dupree, Daniel C. Fabrycky, Debra A. Fischer, Eric B. Ford, Jonathan J. Fortney, Jill Tarter, Forrest R. Girouard, Matthew J. Holman, John Asher Johnson, Todd C. Klaus, Pavel Machalek, Althea V. Moorhead, Robert C. Morehead, Darin Ragozzine, Peter Tenenbaum, Joseph D. Twicken, Samuel N. Quinn, Howard Isaacson, Avi Shporer, Philip W. Lucas, Lucianne M. Walkowicz, William F. Welsh, Alan Boss, Edna Devore, Alan Gould, Jeffrey C. Smith, Robert L. Morris, Andrej Prsa, Timothy D. Morton, Martin Still, Susan E. Thompson, Fergal Mullally, Michael Endl, and Phillip J. MacQueen. Planet Occurrence within 0.25 AU of Solar-type Stars from Kepler. Astrophysical Journal Supplement Series, 201(2):15, August 2012.

[54] Andrew W. Howard, Geoffrey W. Marcy, John Asher Johnson, Debra A. Fischer, Jason T. Wright, Howard Isaacson, Jeff A. Valenti, Jay Anderson, Doug N. C. Lin, and Shigeru Ida. The Occurrence and Mass Distribution of Close-in Super-Earths, Neptunes, and Jupiters. Science, 330(6004):653, October 2010 .

[55] Danley C. Hsu, Eric B. Ford, Darin Ragozzine, and Keir Ashby. Occurrence Rates of Planets Orbiting FGK Stars: Combining Kepler DR25, Gaia DR2, and Bayesian Inference. Astronomical Journal, 158(3):109, September 2019.

[56] Anders Johansen, Melvyn B. Davies, Ross P. Church, and Viktor Holmelin. Can Planetary Instability Explain the Kepler Dichotomy? Astrophysical Journal, 758(1):39, October 2012.

[57] John Asher Johnson, Kimberly M. Aller, Andrew W. Howard, and Justin R. Crepp. Giant Planet Occurrence in the Stellar Mass-Metallicity Plane. Proceedings of the Astronomical Society of the Pacific, 122(894):905, August 2010.

[58] John Asher Johnson, R. Paul Butler, Geoffrey W. Marcy, Debra A. Fischer, Steven S. Vogt, Jason T. Wright, and Kathryn M. G. Peek. A New Planet around an M Dwarf: Revealing a Correlation between Exoplanets and Stellar Mass. Astrophysical Journal, 670(1):833-840, November 2007.

[59] Samson A. Johnson, Matthew Penny, B. Scott Gaudi, Eamonn Kerins, Nicholas J. Rattenbury, Annie C. Robin, Sebastiano Calchi Novati, and Calen B. Henderson. Predictions of the Nancy Grace Roman Space Telescope Galactic Exoplanet Survey. II. Free-floating Planet Detection Rates. Astronomical Journal, 160(3):123, September 2020.

[60] Hugh R. A. Jones, R. Paul Butler, C. G. Tinney, Geoffrey W. Marcy, Brad D. Carter, Alan J. Penny, Chris McCarthy, and Jeremy Bailey. High-eccentricity planets from the Anglo-Australian Planet Search. Monthly Notices of the Royal Astronomical Society, 369(1):249-256, June 2006.

[61] Youn Kil Jung, Andrew Gould, Weicheng Zang, Kyu-Ha Hwang, Yoon-Hyun Ryu, Cheongho Han, Jennifer C. Yee, Michael D. Albrow, Sun-Ju Chung, In-Gu Shin, Yossi Shvartzvald, Wei Zhu, SangMok Cha, Dong-Jin Kim, Hyoun-Woo Kim, Seung-Lee Kim, Chung-Uk Lee, Dong-Joo Lee, Yongseok Lee, Byeong-Gon Park, Richard W. Pogge, KMTNet Collaboration, Matthew T. Penny, Shude Mao, Pascal Fouqué, Tianshu Wang, and CFHT Collaboration. KMT-2017-BLG-0165Lb: A Super-Neptunemass Planet Orbiting a Sun-like Host Star. Astronomical Journal, 157(2):72, February 2019.

[62] M. Kasper, R. Arsenault, U. Käufl, G. Jakob, S. Leveratto, G. Zins, E. Pantin, P. Duhoux, M. Riquelme, J. P. Kirchbauer, J. Kolb, P. Pathak, R. Siebenmorgen, C. Soenke, E. Fuenteseca, M. Sterzik, N. Ageorges, S. Gutruf, D. Kampf, A. Reutlinger, O. Absil, C. Delacroix, A. L. Maire, 
E. Huby, O. Guyon, P. Klupar, D. Mawet, G. Ruane, M. Karlsson, K. Dohlen, A. Vigan, M. N'Diaye, S. Quanz, and A. Carlotti. NEAR: First Results from the Search for Low-Mass Planets in $\alpha$ Cen. The Messenger, 178:5-9, December 2019.

[63] Maciej Konacki, Guillermo Torres, Saurabh Jha, and Dimitar D. Sasselov. An extrasolar planet that transits the disk of its parent star. Nature, 421(6922):507-509, January 2003.

[64] Ravi Kumar Kopparapu, Eric Hébrard, Rus Belikov, Natalie M. Batalha, Gijs D. Mulders, Chris Stark, Dillon Teal, Shawn Domagal-Goldman, and Avi Mandell. Exoplanet Classification and Yield Estimates for Direct Imaging Missions. Astrophysical Journal, 856(2):122, April 2018.

[65] Ravi Kumar Kopparapu, Eric Hébrard, Rus Belikov, Natalie M. Batalha, Gijs D. Mulders, Chris Stark, Dillon Teal, Shawn Domagal-Goldman, and Avi Mandell. Exoplanet Classification and Yield Estimates for Direct Imaging Missions. Astrophysical Journal, 856(2):122, April 2018.

[66] Ravi Kumar Kopparapu, Ramses Ramirez, James F. Kasting, Vincent Eymet, Tyler D. Robinson, Suvrath Mahadevan, Ryan C. Terrien, Shawn Domagal-Goldman, Victoria Meadows, and Rohit Deshpande. Habitable Zones around Main-sequence Stars: New Estimates. Astrophysical Journal, 765(2):131, March 2013.

[67] Ravi Kumar Kopparapu, Ramses M. Ramirez, James SchottelKotte, James F. Kasting, Shawn Domagal-Goldman, and Vincent Eymet. Habitable Zones around Main-sequence Stars: Dependence on Planetary Mass. Astrophysical Journal Letters, 787(2):L29, June 2014.

[68] Kaitlin M. Kratter, Ruth A. Murray-Clay, and Andrew N. Youdin. The Runts of the Litter: Why Planets Formed Through Gravitational Instability Can Only Be Failed Binary Stars. Astrophysical Journal, 710(2):1375-1386, February 2010.

[69] Michelle Kunimoto and Jaymie M. Matthews. Searching the Entirety of Kepler Data. II. Occurrence Rate Estimates for FGK Stars. Astronomical Journal, 159(6):248, June 2020.

[70] David Lafrenière, Ray Jayawardhana, Marten H. van Kerkwijk, Alexis Brandeker, and Markus Janson. An Adaptive Optics Multiplicity Census of Young Stars in Upper Scorpius. Astrophysical Journal, 785(1):47, April 2014.

[71] David W. Latham, Tsevi Mazeh, Robert P. Stefanik, Michel Mayor, and Gilbert Burki. The unseen companion of HD114762: a probable brown dwarf. Nature, 339(6219):38-40, May 1989.

[72] Eve J. Lee and Eugene Chiang. To Cool is to Accrete: Analytic Scalings for Nebular Accretion of Planetary Atmospheres. Astrophysical Journal, 811(1):41, September 2015.

[73] Tim Lichtenberg, Gregor J. Golabek, Remo Burn, Michael R. Meyer, Yann Alibert, Taras V. Gerya, and Christoph Mordasini. A water budget dichotomy of rocky protoplanets from ${ }^{26}$ Al-heating. Nature Astronomy, 3:307-313, February 2019.

[74] D. N. C. Lin, P. Bodenheimer, and D. C. Richardson. Orbital migration of the planetary companion of 51 Pegasi to its present location. Nature, 380(6575):606-607, April 1996.

[75] Jack J. Lissauer, Darin Ragozzine, Daniel C. Fabrycky, Jason H. Steffen, Eric B. Ford, Jon M. Jenkins, Avi Shporer, Matthew J. Holman, Jason F. Rowe, Elisa V. Quintana, Natalie M. Batalha, William J. Borucki, Stephen T. Bryson, Douglas A. Caldwell, Joshua A. Carter, David Ciardi, Edward W. Dunham, Jonathan J. Fortney, III Gautier, Thomas N., Steve B. Howell, David G. Koch, David W. Latham, Geoffrey W. Marcy, Robert C. Morehead, and Dimitar Sasselov. Architecture and Dynamics of Kepler's Candidate Multiple Transiting Planet Systems. Astrophysical Journal Supplement Series, 197(1):8, November 2011.

[76] A. Li Luo, Yong-Heng Zhao, Gang Zhao, Li-Cai Deng, Xiao-Wei Liu, Yi-Peng Jing, Gang Wang, HaoTong Zhang, Jian-Rong Shi, Xiang-Qun Cui, Yao-Quan Chu, Guo-Ping Li, Zhong-Rui Bai, Yue Wu, Yan Cai, Shu-Yun Cao, Zi-Huang Cao, Jeffrey L. Carlin, Hai-Yuan Chen, Jian-Jun Chen, Kun-Xin 
Chen, Li Chen, Xue-Lei Chen, Xiao-Yan Chen, Ying Chen, Norbert Christlieb, Jia-Ru Chu, ChenZhou Cui, Yi-Qiao Dong, Bing Du, Dong-Wei Fan, Lei Feng, Jian-Ning Fu, Peng Gao, Xue-Fei Gong, Bo-Zhong Gu, Yan-Xin Guo, Zhan-Wen Han, Bo-Liang He, Jin-Liang Hou, Yong-Hui Hou, Wen Hou, Hong-Zhuan Hu, Ning-Sheng Hu, Zhong-Wen Hu, Zhi-Ying Huo, Lei Jia, Fang-Hua Jiang, Xiang Jiang, Zhi-Bo Jiang, Ge Jin, Xiao Kong, Xu Kong, Ya-Juan Lei, Ai-Hua Li, Chang-Hua Li, GuangWei Li, Hai-Ning Li, Jian Li, Qi Li, Shuang Li, Sha-Sha Li, Xin-Nan Li, Yan Li, Yin-Bi Li, Ye-Ping Li, Yuan Liang, Chien-Cheng Lin, Chao Liu, Gen-Rong Liu, Guan-Qun Liu, Zhi-Gang Liu, Wen-Zhi Lu, Yu Luo, Yin-Dun Mao, Heidi Newberg, Ji-Jun Ni, Zhao-Xiang Qi, Yong-Jun Qi, Shi-Yin Shen, Huo-Ming Shi, Jing Song, Yi-Han Song, Ding-Qiang Su, Hong-Jun Su, Zheng-Hong Tang, Qing-Sheng Tao, Yuan Tian, Dan Wang, Da-Qi Wang, Feng-Fei Wang, Guo-Min Wang, Hai Wang, Hong-Chi Wang, Jian Wang, Jia-Ning Wang, Jian-Ling Wang, Jian-Ping Wang, Jun-Xian Wang, Lei Wang, Meng-Xin Wang, Shou-Guan Wang, Shu-Qing Wang, Xia Wang, Ya-Nan Wang, You Wang, Yue-Fei Wang, You-Fen Wang, Peng Wei, Ming-Zhi Wei, Hong Wu, Ke-Fei Wu, Xue-Bing Wu, Yu-Zhong Wu, Xiao-Zheng Xing, Ling-Zhe Xu, Xin-Qi Xu, Yan Xu, Tai-Sheng Yan, De-Hua Yang, Hai-Feng Yang, Hui-Qin Yang, Ming Yang, Zheng-Qiu Yao, Yong Yu, Hui Yuan, Hai-Bo Yuan, Hai-Long Yuan, WeiMin Yuan, Chao Zhai, En-Peng Zhang, Hua-Wei Zhang, Jian-Nan Zhang, Li-Pin Zhang, Wei Zhang, Yong Zhang, Yan-Xia Zhang, Zheng-Chao Zhang, Ming Zhao, Fang Zhou, Xu Zhou, Jie Zhu, YongTian Zhu, Si-Cheng Zou, and Fang Zuo. The first data release (DR1) of the LAMOST regular survey. Research in Astronomy and Astrophysics, 15(8):1095, August 2015.

[77] B. Macintosh, J. R. Graham, T. Barman, R. J. De Rosa, Q. Konopacky, M. S. Marley, C. Marois, E. L. Nielsen, L. Pueyo, A. Rajan, J. Rameau, D. Saumon, J. J. Wang, J. Patience, M. Ammons, P. Arriaga, E. Artigau, S. Beckwith, J. Brewster, S. Bruzzone, J. Bulger, B. Burningham, A. S. Burrows, C. Chen, E. Chiang, J. K. Chilcote, R. I. Dawson, R. Dong, R. Doyon, Z. H. Draper, G. Duchêne, T. M. Esposito, D. Fabrycky, M. P. Fitzgerald, K. B. Follette, J. J. Fortney, B. Gerard, S. Goodsell, A. Z. Greenbaum, P. Hibon, S. Hinkley, T. H. Cotten, L. W. Hung, P. Ingraham, M. Johnson-Groh, P. Kalas, D. Lafreniere, J. E. Larkin, J. Lee, M. Line, D. Long, J. Maire, F. Marchis, B. C. Matthews, C. E. Max, S. Metchev, M. A. Millar-Blanchaer, T. Mittal, C. V. Morley, K. M. Morzinski, R. Murray-Clay, R. Oppenheimer, D. W. Palmer, R. Patel, M. D. Perrin, L. A. Poyneer, R. R. Rafikov, F. T. Rantakyrö, E. L. Rice, P. Rojo, A. R. Rudy, J. B. Ruffio, M. T. Ruiz, N. Sadakuni, L. Saddlemyer, M. Salama, D. Savransky, A. C. Schneider, A. Sivaramakrishnan, I. Song, R. Soummer, S. Thomas, G. Vasisht, J. K. Wallace, K. Ward-Duong, S. J. Wiktorowicz, S. G. Wolff, and B. Zuckerman. Discovery and spectroscopy of the young jovian planet 51 Eri b with the Gemini Planet Imager. Science, 350(6256):6467, October 2015.

[78] Fabien Malbet, Alain Léger, Guillem Anglada Escudé, Alessandro Sozzetti, Douglas Spolyar, Lucas Labadie, Mike Shao, Berry Holl, Renaud Goullioud, Antoine Crouzier, Céline Boehm, and Alberto Krone-Martins. Microarcsecond astrometric observatory Theia: from dark matter to compact objects and nearby earths. In Space Telescopes and Instrumentation 2016: Optical, Infrared, and Millimeter Wave, volume 9904 of Society of Photo-Optical Instrumentation Engineers (SPIE) Conference Series, page $99042 \mathrm{~F}$, July 2016.

[79] Jared Males, Laird M. Close, Olivier Guyon, Breann Sitarski, Antonin Bouchez, Alycia Weinberger, and Michael P. Fitzgerald. GMagAO-X: extreme adaptive optics \& coronagraphy for GMT at first light. In Bulletin of the American Astronomical Society, volume 51, page 236, September 2019.

[80] Andrew W. Mann, Eric Gaidos, Sébastien Lépine, and Eric J. Hilton. They Might be Giants: Luminosity Class, Planet Occurrence, and Planet-Metallicity Relation of the Coolest Kepler Target Stars. Astrophysical Journal, 753(1):90, July 2012.

[81] Christian Marois, Bruce Macintosh, Travis Barman, B. Zuckerman, Inseok Song, Jennifer Patience, David Lafrenière, and René Doyon. Direct Imaging of Multiple Planets Orbiting the Star HR 8799. Science, 322(5906):1348, November 2008.

[82] E. Masciadri, R. Mundt, Th. Henning, C. Alvarez, and D. Barrado y Navascués. A Search for Hot Massive Extrasolar Planets around Nearby Young Stars with the Adaptive Optics System NACO. Astrophysical Journal, 625(2):1004-1018, June 2005. 
[83] Dimitri Mawet, Lea Hirsch, Eve J. Lee, Jean-Baptiste Ruffio, Michael Bottom, Benjamin J. Fulton, Olivier Absil, Charles Beichman, Brendan Bowler, Marta Bryan, Elodie Choquet, David Ciardi, Valentin Christiaens, Denis Defrère, Carlos Alberto Gomez Gonzalez, Andrew W. Howard, Elsa Huby, Howard Isaacson, Rebecca Jensen-Clem, Molly Kosiarek, Geoff Marcy, Tiffany Meshkat, Erik Petigura, Maddalena Reggiani, Garreth Ruane, Eugene Serabyn, Evan Sinukoff, Ji Wang, Lauren Weiss, and Marie Ygouf. Deep Exploration of $\in$ Eridani with Keck Ms-band Vortex Coronagraphy and Radial Velocities: Mass and Orbital Parameters of the Giant Exoplanet. Astronomical Journal, 157(1):33, January 2019.

[84] M. Mayor, M. Marmier, C. Lovis, S. Udry, D. Ségransan, F. Pepe, W. Benz, J. L. Bertaux, F. Bouchy, X. Dumusque, G. Lo Curto, C. Mordasini, D. Queloz, and N. C. Santos. The HARPS search for southern extra-solar planets XXXIV. Occurrence, mass distribution and orbital properties of superEarths and Neptune-mass planets. arXiv e-prints, page arXiv:1109.2497, September 2011.

[85] Michel Mayor and Didier Queloz. A Jupiter-mass companion to a solar-type star. Nature, 378(6555):355-359, November 1995.

[86] T. Mazeh, T. Holczer, and S. Faigler. Dearth of short-period Neptunian exoplanets: A desert in period-mass and period-radius planes. Astronomy \& Astrophysics, 589:A75, May 2016.

[87] Stanimir A. Metchev and Lynne A. Hillenbrand. The Palomar/Keck Adaptive Optics Survey of Young Solar Analogs: Evidence for a Universal Companion Mass Function. Astrophysical Journal Supplement Series, 181(1):62-109, March 2009.

[88] Michael R. Meyer, Adam Amara, Maddalena Reggiani, and Sascha P. Quanz. M-dwarf exoplanet surface density distribution. A log-normal fit from 0.07 to $400 \mathrm{AU}$. Astronomy \& Astrophysics, 612:L3, April 2018.

[89] H. Mizuno. Formation of the Giant Planets. Progress of Theoretical Physics, 64(2):544-557, August 1980.

[90] B. J. Mochejska, K. Z. Stanek, D. D. Sasselov, A. H. Szentgyorgyi, G. Á. Bakos, J. Hradecky, V. Devor, D. P. Marrone, J. N. Winn, and M. Zaldarriaga. Planets in Stellar Clusters Extensive Search. III. A Search for Transiting Planets in the Metal-rich Open Cluster NGC 6791. Astronomical Journal, 129(6):2856-2868, June 2005.

[91] A. Morbidelli, B. Bitsch, A. Crida, M. Gounelle, T. Guillot, S. Jacobson, A. Johansen, M. Lambrechts, and E. Lega. Fossilized condensation lines in the Solar System protoplanetary disk. Icarus, 267:368-376, March 2016.

[92] Gijs D. Mulders, Ilaria Pascucci, Dániel Apai, and Fred J. Ciesla. The Exoplanet Population Observation Simulator. I. The Inner Edges of Planetary Systems. Astronomical Journal, 156(1):24, July 2018.

[93] Lena Murchikova and Scott Tremaine. Peas in a Pod? Radius Correlations in Kepler Multiplanet Systems. Astronomical Journal, 160(4):160, October 2020.

[94] Eric L. Nielsen and Laird M. Close. A Uniform Analysis of 118 Stars with High-contrast Imaging: Longperiod Extrasolar Giant Planets are Rare Around Sun-like Stars. Astrophysical Journal, 717(2):878896, July 2010.

[95] Eric L. Nielsen, Robert J. De Rosa, Bruce Macintosh, Jason J. Wang, Jean-Baptiste Ruffio, Eugene Chiang, Mark S. Marley, Didier Saumon, Dmitry Savransky, S. Mark Ammons, Vanessa P. Bailey, Travis Barman, Célia Blain, Joanna Bulger, Adam Burrows, Jeffrey Chilcote, Tara Cotten, Ian Czekala, Rene Doyon, Gaspard Duchêne, Thomas M. Esposito, Daniel Fabrycky, Michael P. Fitzgerald, Katherine B. Follette, Jonathan J. Fortney, Benjamin L. Gerard, Stephen J. Goodsell, James R. Graham, Alexandra Z. Greenbaum, Pascale Hibon, Sasha Hinkley, Lea A. Hirsch, Justin Hom, Li-Wei Hung, Rebekah Ilene Dawson, Patrick Ingraham, Paul Kalas, Quinn Konopacky, James E. Larkin, 
Eve J. Lee, Jonathan W. Lin, Jérôme Maire, Franck Marchis, Christian Marois, Stanimir Metchev, Maxwell A. Millar-Blanchaer, Katie M. Morzinski, Rebecca Oppenheimer, David Palmer, Jennifer Patience, Marshall Perrin, Lisa Poyneer, Laurent Pueyo, Roman R. Rafikov, Abhijith Rajan, Julien Rameau, Fredrik T. Rantakyrö, Bin Ren, Adam C. Schneider, Anand Sivaramakrishnan, Inseok Song, Remi Soummer, Melisa Tallis, Sandrine Thomas, Kimberly Ward-Duong, and Schuyler Wolff. The Gemini Planet Imager Exoplanet Survey: Giant Planet and Brown Dwarf Demographics from 10 to $100 \mathrm{au}$. Astronomical Journal, 158(1):13, July 2019.

[96] National Academies of Sciencesd. Exoplanet Science Strategy. The National Academies Press, Washington, DC, 2018.

[97] E. Öpik. Statistical Studies of Double Stars: On the Distribution of Relative Luminosities and Distances of Double Stars in the Harvard Revised Photometry North of Declination $-31^{\circ}$. Publications of the Tartu Astrofizica Observatory, 25:1, January 1924.

[98] Ilaria Pascucci, Gijs D. Mulders, and Eric Lopez. The Impact of Stripped Cores on the Frequency of Earth-size Planets in the Habitable Zone. Astrophysical Journal Letters, 883(1):L15, September 2019.

[99] Matthew T. Penny, B. Scott Gaudi, Eamonn Kerins, Nicholas J. Rattenbury, Shude Mao, Annie C. Robin, and Sebastiano Calchi Novati. Predictions of the WFIRST Microlensing Survey. I. Bound Planet Detection Rates. Astrophysical Journal Supplement Series, 241(1):3, March 2019.

[100] Michael Perryman, Joel Hartman, Gáspár Á. Bakos, and Lennart Lindegren. Astrometric Exoplanet Detection with Gaia. Astrophysical Journal, 797(1):14, December 2014.

[101] Erik A. Petigura, Andrew W. Howard, and Geoffrey W. Marcy. Prevalence of Earth-size planets orbiting Sun-like stars. Proceedings of the National Academy of Science, 110(48):19273-19278, November 2013.

[102] Erik A. Petigura, Geoffrey W. Marcy, Joshua N. Winn, Lauren M. Weiss, Benjamin J. Fulton, Andrew W. Howard, Evan Sinukoff, Howard Isaacson, Timothy D. Morton, and John Asher Johnson. The California-Kepler Survey. IV. Metal-rich Stars Host a Greater Diversity of Planets. Astronomical Journal, 155(2):89, February 2018.

[103] Peter Plavchan, Dave Latham, Scott Gaudi, Justin Crepp, Xavier Dumusque, Gabor Furesz, Andrew Vand erburg, Cullen Blake, Debra Fischer, Lisa Prato, Russel White, Valeri Makarov, Geoff Marcy, Karl Stapelfeldt, Raphaëlle Haywood, Andrew Collier-Cameron, Andreas Quirrenbach, Suvrath Mahadevan, Guillem Anglada, and Philip Muirhead. Radial Velocity Prospects Current and Future: A White Paper Report prepared by the Study Analysis Group 8 for the Exoplanet Program Analysis Group (ExoPAG). arXiv e-prints, page arXiv:1503.01770, March 2015.

[104] James B. Pollack, Olenka Hubickyj, Peter Bodenheimer, Jack J. Lissauer, Morris Podolak, and Yuval Greenzweig. Formation of the Giant Planets by Concurrent Accretion of Solids and Gas. Icarus, 124(1):62-85, November 1996.

[105] Sascha Quanz. The LIFE mission: a space mission designed to characterize terrestrial exoplanet atmospheres. In EPSC-DPS Joint Meeting 2019, volume 2019, pages EPSC-DPS2019-327, September 2019.

[106] Roman R. Rafikov. Can Giant Planets Form by Direct Gravitational Instability? Astrophysical Journal Letters, 621(1):L69-L72, March 2005.

[107] Roman R. Rafikov. Atmospheres of Protoplanetary Cores: Critical Mass for Nucleated Instability. Astrophysical Journal, 648(1):666-682, September 2006.

[108] Frederic A. Rasio and Eric B. Ford. Dynamical instabilities and the formation of extrasolar planetary systems. Science, 274:954-956, November 1996. 
[109] H. Rauer, C. Catala, C. Aerts, T. Appourchaux, W. Benz, A. Brandeker, J. Christensen-Dalsgaard, M. Deleuil, L. Gizon, M. J. Goupil, M. Güdel, E. Janot-Pacheco, M. Mas-Hesse, I. Pagano, G. Piotto, D. Pollacco, C. Santos, A. Smith, J. C. Suárez, R. Szabó, S. Udry, V. Adibekyan, Y. Alibert, J. M. Almenara, P. Amaro-Seoane, M. Ammler-von Eiff, M. Asplund, E. Antonello, S. Barnes, F. Baudin, K. Belkacem, M. Bergemann, G. Bihain, A. C. Birch, X. Bonfils, I. Boisse, A. S. Bonomo, F. Borsa, I. M. Brand ão, E. Brocato, S. Brun, M. Burleigh, R. Burston, J. Cabrera, S. Cassisi, W. Chaplin, S. Charpinet, C. Chiappini, R. P. Church, Sz. Csizmadia, M. Cunha, M. Damasso, M. B. Davies, H. J. Deeg, R. F. Díaz, S. Dreizler, C. Dreyer, P. Eggenberger, D. Ehrenreich, P. Eigmüller, A. Erikson, R. Farmer, S. Feltzing, F. de Oliveira Fialho, P. Figueira, T. Forveille, M. Fridlund, R. A. García, P. Giommi, G. Giuffrida, M. Godolt, J. Gomes da Silva, T. Granzer, J. L. Grenfell, A. GrotschNoels, E. Günther, C. A. Haswell, A. P. Hatzes, G. Hébrard, S. Hekker, R. Helled, K. Heng, J. M. Jenkins, A. Johansen, M. L. Khodachenko, K. G. Kislyakova, W. Kley, U. Kolb, N. Krivova, F. Kupka, H. Lammer, A. F. Lanza, Y. Lebreton, D. Magrin, P. Marcos-Arenal, P. M. Marrese, J. P. Marques, J. Martins, S. Mathis, S. Mathur, S. Messina, A. Miglio, J. Montalban, M. Montalto, M. J. P. F. G. Monteiro, H. Moradi, E. Moravveji, C. Mordasini, T. Morel, A. Mortier, V. Nascimbeni, R. P. Nelson, M. B. Nielsen, L. Noack, A. J. Norton, A. Ofir, M. Oshagh, R. M. Ouazzani, P. Pápics, V. C. Parro, P. Petit, B. Plez, E. Poretti, A. Quirrenbach, R. Ragazzoni, G. Raimondo, M. Rainer, D. R. Reese, R. Redmer, S. Reffert, B. Rojas-Ayala, I. W. Roxburgh, S. Salmon, A. Santerne, J. Schneider, J. Schou, S. Schuh, H. Schunker, A. Silva-Valio, R. Silvotti, I. Skillen, I. Snellen, F. Sohl, S. G. Sousa, A. Sozzetti, D. Stello, K. G. Strassmeier, M. Švanda, Gy. M. Szabó, A. Tkachenko, D. Valencia, V. Van Grootel, S. D. Vauclair, P. Ventura, F. W. Wagner, N. A. Walton, J. Weingrill, S. C. Werner, P. J. Wheatley, and K. Zwintz. The PLATO 2.0 mission. Experimental Astronomy, 38(1-2):249-330, November 2014.

[110] Sean N. Raymond and Alessandro Morbidelli. Planet formation: key mechanisms and global models. arXiv e-prints, page arXiv:2002.05756, February 2020.

[111] M. Reggiani, M. R. Meyer, G. Chauvin, A. Vigan, S. P. Quanz, B. Biller, M. Bonavita, S. Desidera, P. Delorme, J. Hagelberg, A. L. Maire, A. Boccaletti, J. L. Beuzit, E. Buenzli, J. Carson, E. Covino, M. Feldt, J. Girard, R. Gratton, T. Henning, M. Kasper, A. M. Lagrange, D. Mesa, S. Messina, G. Montagnier, C. Mordasini, D. Mouillet, J. E. Schlieder, D. Segransan, C. Thalmann, and A. Zurlo. The VLT/NaCo large program to probe the occurrence of exoplanets and brown dwarfs at wide orbits . III. The frequency of brown dwarfs and giant planets as companions to solar-type stars. Astronomy \& Astrophysics, 586:A147, February 2016.

[112] George R. Ricker, Joshua N. Winn, Roland Vanderspek, David W. Latham, Gáspár Á. Bakos, Jacob L. Bean, Zachory K. Berta-Thompson, Timothy M. Brown, Lars Buchhave, Nathaniel R. Butler, R. Paul Butler, William J. Chaplin, David Charbonneau, Jørgen Christensen-Dalsgaard, Mark Clampin, Drake Deming, John Doty, Nathan De Lee, Courtney Dressing, Edward W. Dunham, Michael Endl, Francois Fressin, Jian Ge, Thomas Henning, Matthew J. Holman, Andrew W. Howard, Shigeru Ida, Jon M. Jenkins, Garrett Jernigan, John Asher Johnson, Lisa Kaltenegger, Nobuyuki Kawai, Hans Kjeldsen, Gregory Laughlin, Alan M. Levine, Douglas Lin, Jack J. Lissauer, Phillip MacQueen, Geoffrey Marcy, Peter R. McCullough, Timothy D. Morton, Norio Narita, Martin Paegert, Enric Palle, Francesco Pepe, Joshua Pepper, Andreas Quirrenbach, Stephen A. Rinehart, Dimitar Sasselov, Bun'ei Sato, Sara Seager, Alessandro Sozzetti, Keivan G. Stassun, Peter Sullivan, Andrew Szentgyorgyi, Guillermo Torres, Stephane Udry, and Joel Villasenor. Transiting Exoplanet Survey Satellite (TESS). Journal of Astronomical Telescopes, Instruments, and Systems, 1:014003, January 2015.

[113] Leslie A. Rogers. Most 1.6 Earth-radius Planets are Not Rocky. Astrophysical Journal, 801(1):41, March 2015.

[114] M. M. Rosenthal, E. I. Chiang, S. Ginzburg, and R. A. Murray-Clay. How consumption and repulsion set planetary gap depths and the final masses of gas giants. Monthly Notices of the Royal Astronomical Society, 498(2):2054-2067, June 2020.

[115] M. M. Rosenthal and R. A. Murray-Clay. How Flow Isolation May Set the Mass Scale for Super-Earth Planets. Astrophysical Journal, 898(2):108, August 2020. 
[116] Kevin C. Schlaufman. Evidence of an Upper Bound on the Masses of Planets and Its Implications for Giant Planet Formation. Astrophysical Journal, 853(1):37, January 2018.

[117] I. A. G. Snellen and A. G. A. Brown. The mass of the young planet Beta Pictoris b through the astrometric motion of its host star. Nature Astronomy, 2:883-886, August 2018.

[118] Alessandro Sozzetti, Guillermo Torres, David W. Latham, Robert P. Stefanik, Sylvain G. Korzennik, Alan P. Boss, Bruce W. Carney, and John B. Laird. A Keck HIRES Doppler Search for Planets Orbiting Metal-Poor Dwarfs. II. On the Frequency of Giant Planets in the Metal-Poor Regime. Astrophysical Journal, 697(1):544-556, May 2009.

[119] D. Spergel, N. Gehrels, C. Baltay, D. Bennett, J. Breckinridge, M. Donahue, A. Dressler, B. S. Gaudi, T. Greene, O. Guyon, C. Hirata, J. Kalirai, N. J. Kasdin, B. Macintosh, W. Moos, S. Perlmutter, M. Postman, B. Rauscher, J. Rhodes, Y. Wang, D. Weinberg, D. Benford, M. Hudson, W. S. Jeong, Y. Mellier, W. Traub, T. Yamada, P. Capak, J. Colbert, D. Masters, M. Penny, D. Savransky, D. Stern, N. Zimmerman, R. Barry, L. Bartusek, K. Carpenter, E. Cheng, D. Content, F. Dekens, R. Demers, K. Grady, C. Jackson, G. Kuan, J. Kruk, M. Melton, B. Nemati, B. Parvin, I. Poberezhskiy, C. Peddie, J. Ruffa, J. K. Wallace, A. Whipple, E. Wollack, and F. Zhao. Wide-Field InfrarRed Survey Telescope-Astrophysics Focused Telescope Assets WFIRST-AFTA 2015 Report. arXiv e-prints, page arXiv:1503.03757, March 2015.

[120] D. Suzuki, D. P. Bennett, T. Sumi, I. A. Bond, L. A. Rogers, F. Abe, Y. Asakura, A. Bhattacharya, M. Donachie, M. Freeman, A. Fukui, Y. Hirao, Y. Itow, N. Koshimoto, M. C. A. Li, C. H. Ling, K. Masuda, Y. Matsubara, Y. Muraki, M. Nagakane, K. Onishi, H. Oyokawa, N. Rattenbury, To. Saito, A. Sharan, H. Shibai, D. J. Sullivan, P. J. Tristram, A. Yonehara, and MOA Collaboration. The Exoplanet Mass-ratio Function from the MOA-II Survey: Discovery of a Break and Likely Peak at a Neptune Mass. Astrophysical Journal, 833(2):145, December 2016.

[121] Jonathan J. Swift, John Asher Johnson, Timothy D. Morton, Justin R. Crepp, Benjamin T. Montet, Daniel C. Fabrycky, and Philip S. Muirhead. Characterizing the Cool KOIs. IV. Kepler-32 as a Prototype for the Formation of Compact Planetary Systems throughout the Galaxy. Astrophysical Journal, 764(1):105, February 2013.

[122] The LUVOIR Team. The LUVOIR Mission Concept Study Final Report. arXiv e-prints, page arXiv:1912.06219, December 2019.

[123] A. Udalski, Y. H. Ryu, S. Sajadian, A. Gould, P. Mróz, R. Poleski, M. K. Szymański, J. Skowron, I. Soszyński, S. Kozłowski, P. Pietrukowicz, K. Ulaczyk, M. Pawlak, K. Rybicki, P. Iwanek, M. D. Albrow, S. J. Chung, C. Han, K. H. Hwang, K. Jung, Y., I. G. Shin, Y. Shvartzvald, J. C. Yee, W. Zang, W. Zhu, S. M. Cha, D. J. Kim, H. W. Kim, S. L. Kim, C. U. Lee, D. J. Lee, Y. Lee, B. G. Park, R. W. Pogge, V. Bozza, M. Dominik, C. Helling, M. Hundertmark, U. G. Jørgensen, P. LongaPeña, S. Lowry, M. Burgdorf, J. Campbell-White, S. Ciceri, D. Evans, R. Figuera Jaimes, Y. I. Fujii, L. K. Haikala, T. Henning, T. C. Hinse, L. Mancini, N. Peixinho, S. Rahvar, M. Rabus, J. Skottfelt, C. Snodgrass, J. Southworth, and C. von Essen. OGLE-2017-BLG-1434Lb: Eighth $q<1 \times 10^{-4}$ Mass-Ratio Microlens Planet Confirms Turnover in Planet Mass-Ratio Function. Acta Astronomica, 68(1):1-42, March 2018.

[124] A. Udalski, K. Zebrun, M. Szymanski, M. Kubiak, I. Soszynski, O. Szewczyk, L. Wyrzykowski, and G. Pietrzynski. The Optical Gravitational Lensing Experiment. Search for Planetary and Low- Luminosity Object Transits in the Galactic Disk. Results of 2001 Campaign - Supplement. Acta Astronomica, 52:115-128, June 2002.

[125] Aikaterini Vandorou, David P. Bennett, Jean-Philippe Beaulieu, Christophe Alard, Joshua W. Blackman, Andrew A. Cole, Aparna Bhattacharya, Ian A. Bond, Naoki Koshimoto, and Jean-Baptiste Marquette. Revisiting MOA 2013-BLG-220L: A Solar-type Star with a Cold Super-Jupiter Companion. Astronomical Journal, 160(3):121, September 2020. 
[126] Dimitri Veras, Mark C. Wyatt, Alexander J. Mustill, Amy Bonsor, and John J. Eldridge. The great escape: how exoplanets and smaller bodies desert dying stars. Monthly Notices of the Royal Astronomical Society, 417(3):2104-2123, November 2011.

[127] A. Vigan, M. Bonavita, B. Biller, D. Forgan, K. Rice, G. Chauvin, S. Desidera, J. C. Meunier, P. Delorme, J. E. Schlieder, M. Bonnefoy, J. Carson, E. Covino, J. Hagelberg, T. Henning, M. Janson, A. M. Lagrange, S. P. Quanz, A. Zurlo, J. L. Beuzit, A. Boccaletti, E. Buenzli, M. Feldt, J. H. V. Girard, R. Gratton, M. Kasper, H. Le Coroller, D. Mesa, S. Messina, M. Meyer, G. Montagnier, C. Mordasini, D. Mouillet, C. Moutou, M. Reggiani, D. Segransan, and C. Thalmann. The VLT/NaCo large program to probe the occurrence of exoplanets and brown dwarfs at wide orbits. IV. Gravitational instability rarely forms wide, giant planets. Astronomy \& Astrophysics, 603:A3, June 2017.

[128] A. Vigan, C. Fontanive, M. Meyer, B. Biller, M. Bonavita, M. Feldt, S. Desidera, G. D. Marleau, A. Emsenhuber, R. Galicher, K. Rice, D. Forgan, C. Mordasini, R. Gratton, H. Le Coroller, A. L. Maire, F. Cantalloube, G. Chauvin, A. Cheetham, J. Hagelberg, A. M. Lagrange, M. Langlois, M. Bonnefoy, J. L. Beuzit, A. Boccaletti, V. D'Orazi, P. Delorme, C. Dominik, Th. Henning, M. Janson, E. Lagadec, C. Lazzoni, R. Ligi, F. Menard, D. Mesa, S. Messina, C. Moutou, A. Müller, C. Perrot, M. Samland, H. M. Schmid, T. Schmidt, E. Sissa, M. Turatto, S. Udry, A. Zurlo, L. Abe, J. Antichi, R. AsensioTorres, A. Baruffolo, P. Baudoz, J. Baudrand, A. Bazzon, P. Blanchard, A. J. Bohn, S. Brown Sevilla, M. Carbillet, M. Carle, E. Cascone, J. Charton, R. Claudi, A. Costille, V. De Caprio, A. Delboulbé, K. Dohlen, N. Engler, D. Fantinel, P. Feautrier, T. Fusco, P. Gigan, J. H. Girard, E. Giro, D. Gisler, L. Gluck, C. Gry, N. Hubin, E. Hugot, M. Jaquet, M. Kasper, D. Le Mignant, M. Llored, F. Madec, Y. Magnard, P. Martinez, D. Maurel, O. Möller-Nilsson, D. Mouillet, T. Moulin, A. Origné, A. Pavlov, D. Perret, C. Petit, J. Pragt, P. Puget, P. Rabou, J. Ramos, E. L. Rickman, F. Rigal, S. Rochat, R. Roelfsema, G. Rousset, A. Roux, B. Salasnich, J. F. Sauvage, A. Sevin, C. Soenke, E. Stadler, M. Suarez, Z. Wahhaj, L. Weber, and F. Wildi. The SPHERE infrared survey for exoplanets (SHINE). III. The demographics of young giant exoplanets below 300 au with SPHERE. arXiv e-prints, page arXiv:2007.06573, July 2020.

[129] Ji Wang, Michael Meyer, Alan Boss, Laird Close, Thayne Currie, Diana Dragomir, Jonathan Fortney, Eric Gaidos, Yasuhiro Hasegawa, Irina Kitiashvili, Quinn Konopacky, Chien-Hsiu Lee, Nikole K. Lewis, Michael Liu, Roxana Lupu, Dimitri Mawet, Carl Melis, Mercedes Lopez-Morales, Caroline V. Morley, Chris Packham, Eliad Peretz, Andy Skemer, and Mel Ulmer. New Frontiers for Terrestrial-sized to Neptune-sized Exoplanets In the Era of Extremely Large Telescopes. Bulletin of the American Astronomical Society, 51(3):200, May 2019.

[130] Lauren M. Weiss, Geoffrey W. Marcy, Erik A. Petigura, Benjamin J. Fulton, Andrew W. Howard, Joshua N. Winn, Howard T. Isaacson, Timothy D. Morton, Lea A. Hirsch, Evan J. Sinukoff, Andrew Cumming, Leslie Hebb, and Phillip A. Cargile. The California-Kepler Survey. V. Peas in a Pod: Planets in a Kepler Multi-planet System Are Similar in Size and Regularly Spaced. Astronomical Journal, 155(1):48, January 2018.

[131] Lauren M. Weiss and Erik A. Petigura. The Kepler Peas in a Pod Pattern is Astrophysical. Astrophysical Journal Letters, 893(1):L1, April 2020.

[132] David T. F. Weldrake, Penny D. Sackett, and Terry J. Bridges. The Frequency of Large-Radius Hot and Very Hot Jupiters in $\omega$ Centauri. Astrophysical Journal, 674(2):1117-1129, February 2008.

[133] David T. F. Weldrake, Penny D. Sackett, Terry J. Bridges, and Kenneth C. Freeman. An Absence of Hot Jupiter Planets in 47 Tucanae: Results of a Wide-Field Transit Search. Astrophysical Journal, 620(2):1043-1051, February 2005.

[134] Angie Wolfgang, Leslie A. Rogers, and Eric B. Ford. Probabilistic Mass-Radius Relationship for SubNeptune-Sized Planets. Astrophysical Journal, 825(1):19, July 2016.

[135] A. Wolszczan and D. A. Frail. A planetary system around the millisecond pulsar PSR1257 + 12. Nature, 355(6356):145-147, January 1992. 
[136] Yuhan Yao, Michael R. Meyer, Kevin R. Covey, Jonathan C. Tan, and Nicola Da Rio. IN-SYNC. VIII. Primordial Disk Frequencies in NGC 1333, IC 348, and the Orion A Molecular Cloud. Astrophysical Journal, 869(1):72, December 2018.

[137] Andrew N. Youdin. The Exoplanet Census: A General Method Applied to Kepler. Astrophysical Journal, 742(1):38, November 2011.

[138] Wei Zhu. On the Patterns Observed in Kepler Multi-planet Systems. Astronomical Journal, 159(5):188, May 2020.

[139] Wei Zhu, Ji Wang, and Chelsea Huang. Dependence of Small Planet Frequency on Stellar Metallicity Hidden by Their Prevalence. Astrophysical Journal, 832(2):196, December 2016.

[140] Jon K. Zink and Bradley M. S. Hansen. Accounting for multiplicity in calculating eta Earth. Monthly Notices of the Royal Astronomical Society, 487(1):246-252, July 2019. 